\title{
Discovery of N-Alkyl Catecholamides as Selective \\ Phosphodiesterase-4 Inhibitors with Anti-neuroinflammation Exhibiting Antidepressant-like Effects at Non-emetic Doses
}

\author{
Zhong-Zhen Zhou ${ }^{\text {a, *, Yu-Fang Cheng }}{ }^{\text {a }}$, Zheng-Qiang Zou ${ }^{\text {a }}$, Bing-Chen Ge ${ }^{\text {a }}$, Hui Yu ${ }^{\text {a }}$, Cang Huang ${ }^{\text {a }}$, \\ Hai-Tao Wang ${ }^{a}$, Xue-Mei Yang ${ }^{\mathrm{b}, *}$ and Jiang-Ping $\mathrm{Xu}^{\mathrm{a}, *}$ \\ a Neuropharmacology Group, School of Pharmaceutical Sciences, Southern Medical University, \\ Guangzhou 510515, China. Fax: +86 20 61648235; \\ ${ }^{\mathrm{b}}$ Hygiene Detection Center, School of Public Health, Southern Medical University, Guangzhou 510515, \\ China. \\ * Corresponding authors. E-mail addresses: zhouzz@smu.edu.cn (Z.-Z Zhou), yxmjp@sina.com (X.-M. \\ Yang),jpx@smu.edu.cn (J.-P. Xu).
}

\section{Supporting Information}

1. Figures $\mathrm{S} 1 \sim 16 .{ }^{1} \mathrm{H}$ and ${ }^{13} \mathrm{C}$ NMR spectra of compounds $10 \mathbf{a}-\mathbf{p} . . . . . . . . . . . . . . S 2-\mathrm{S} 17$ 


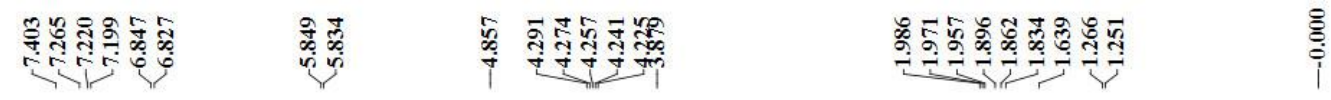
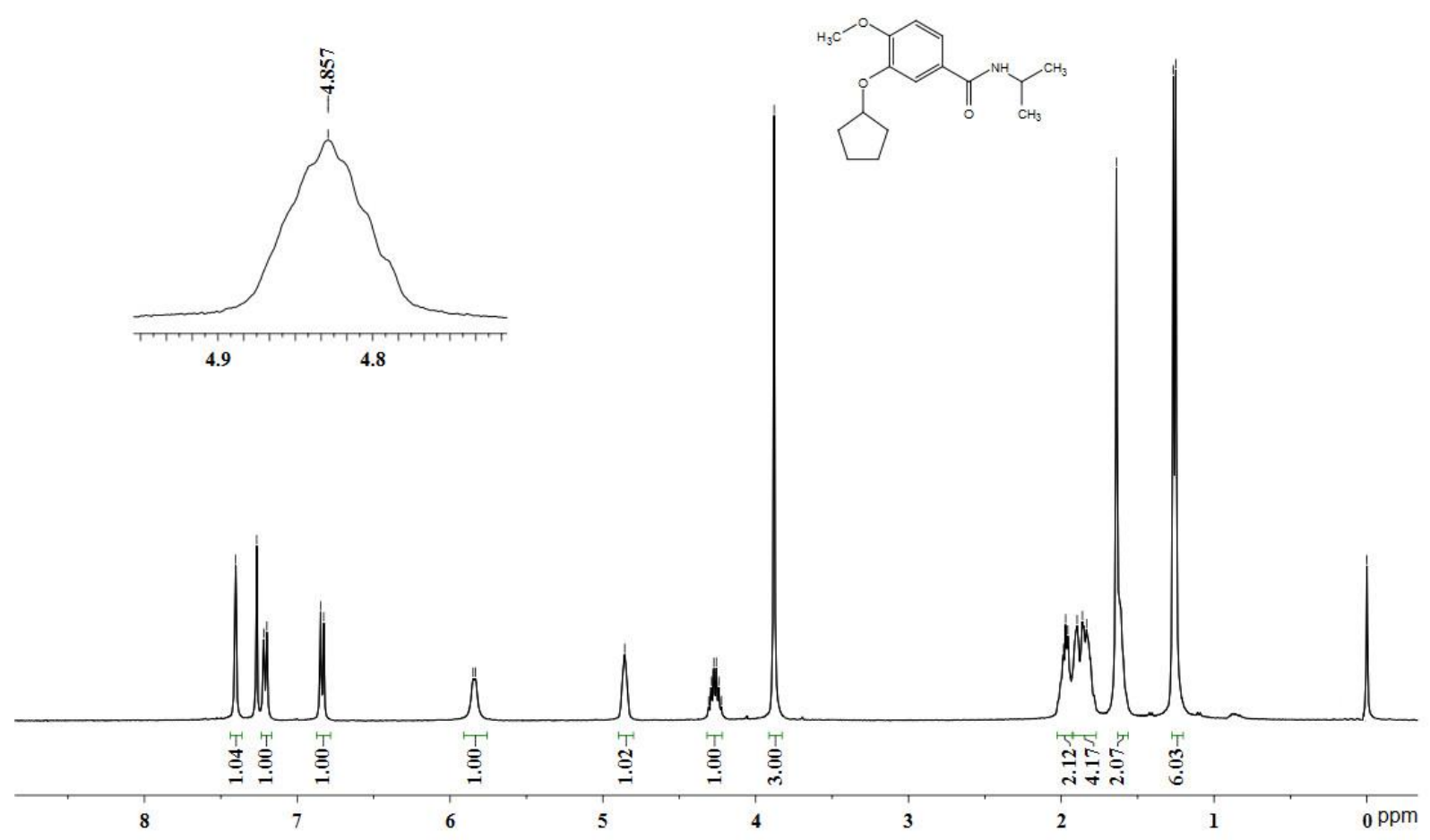

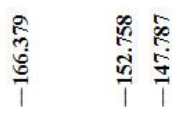

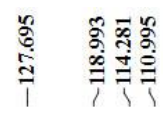

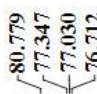

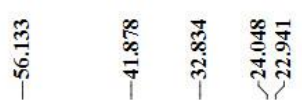
$\stackrel{\overbrace{}}{\stackrel{\text { g }}{i}}$
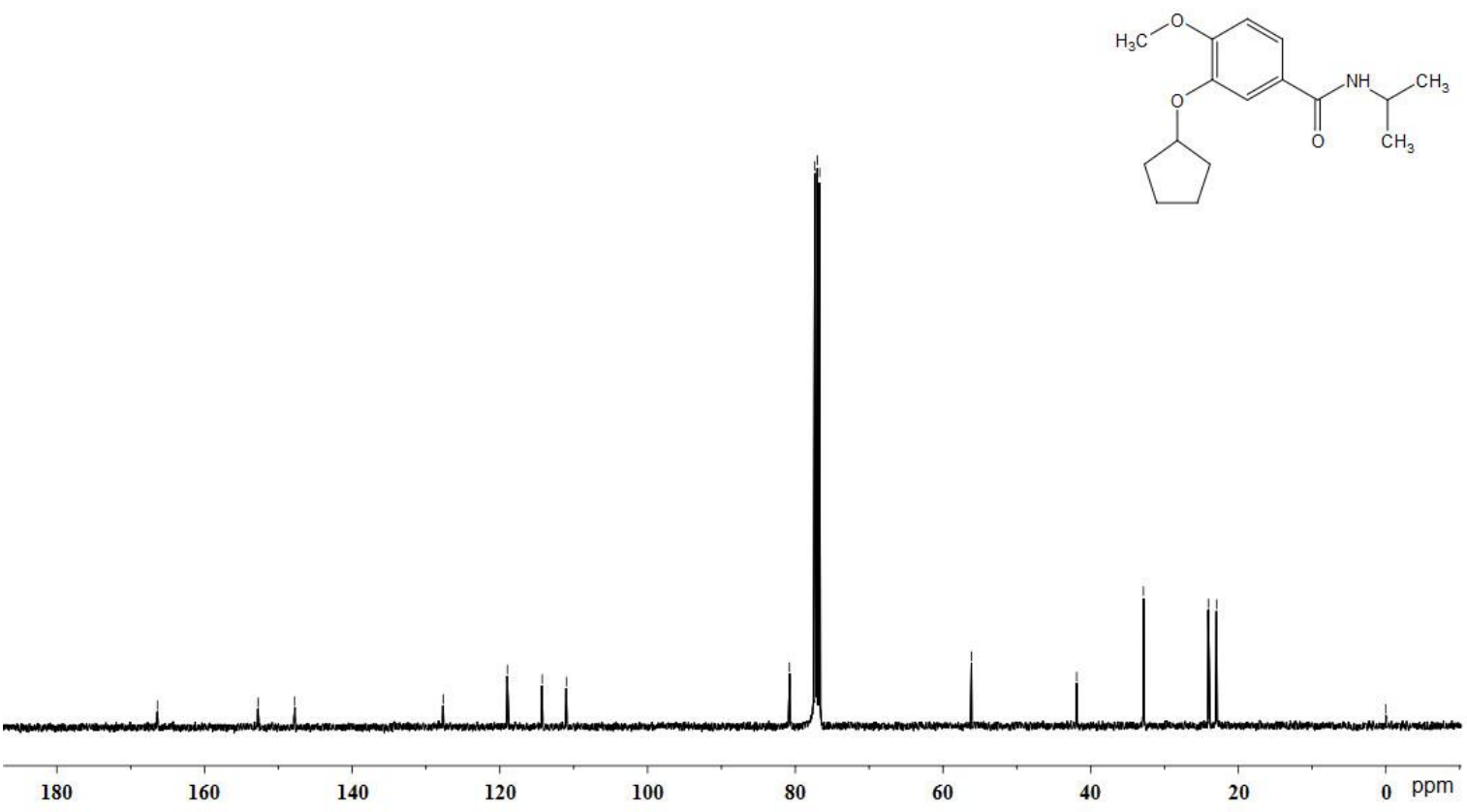

Figure S1. ${ }^{1} \mathrm{H}-\mathrm{NMR}\left(\mathrm{CDCl}_{3,}, 400 \mathrm{MHz}\right)$ and ${ }^{13} \mathrm{C}-\mathrm{NMR}\left(\mathrm{CDCl}_{3}, 101 \mathrm{MHz}\right)$ spectra of compound 10a 


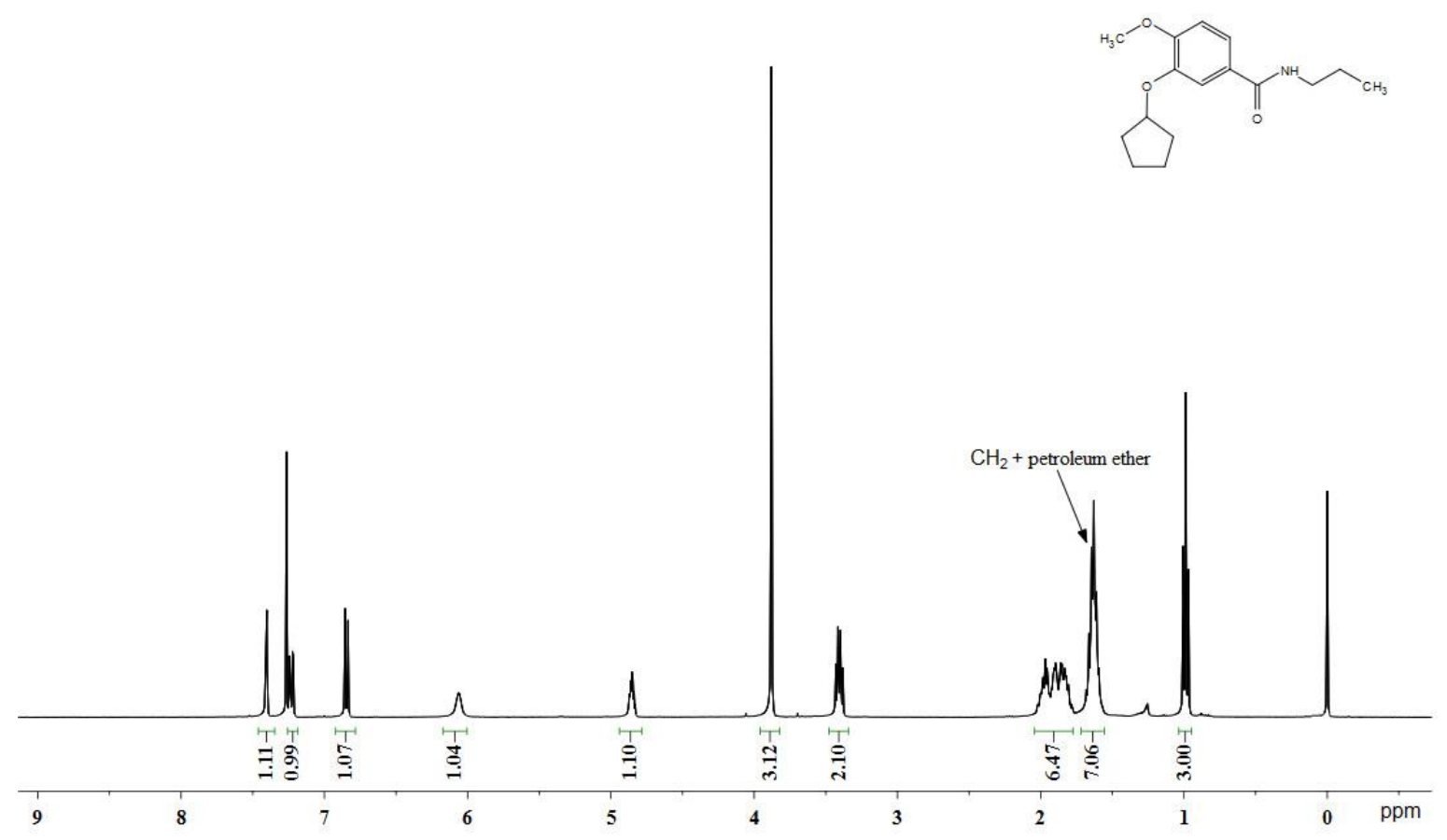

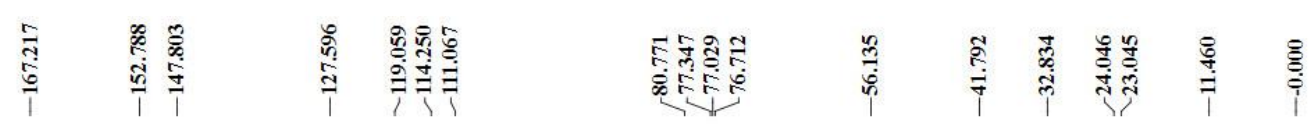

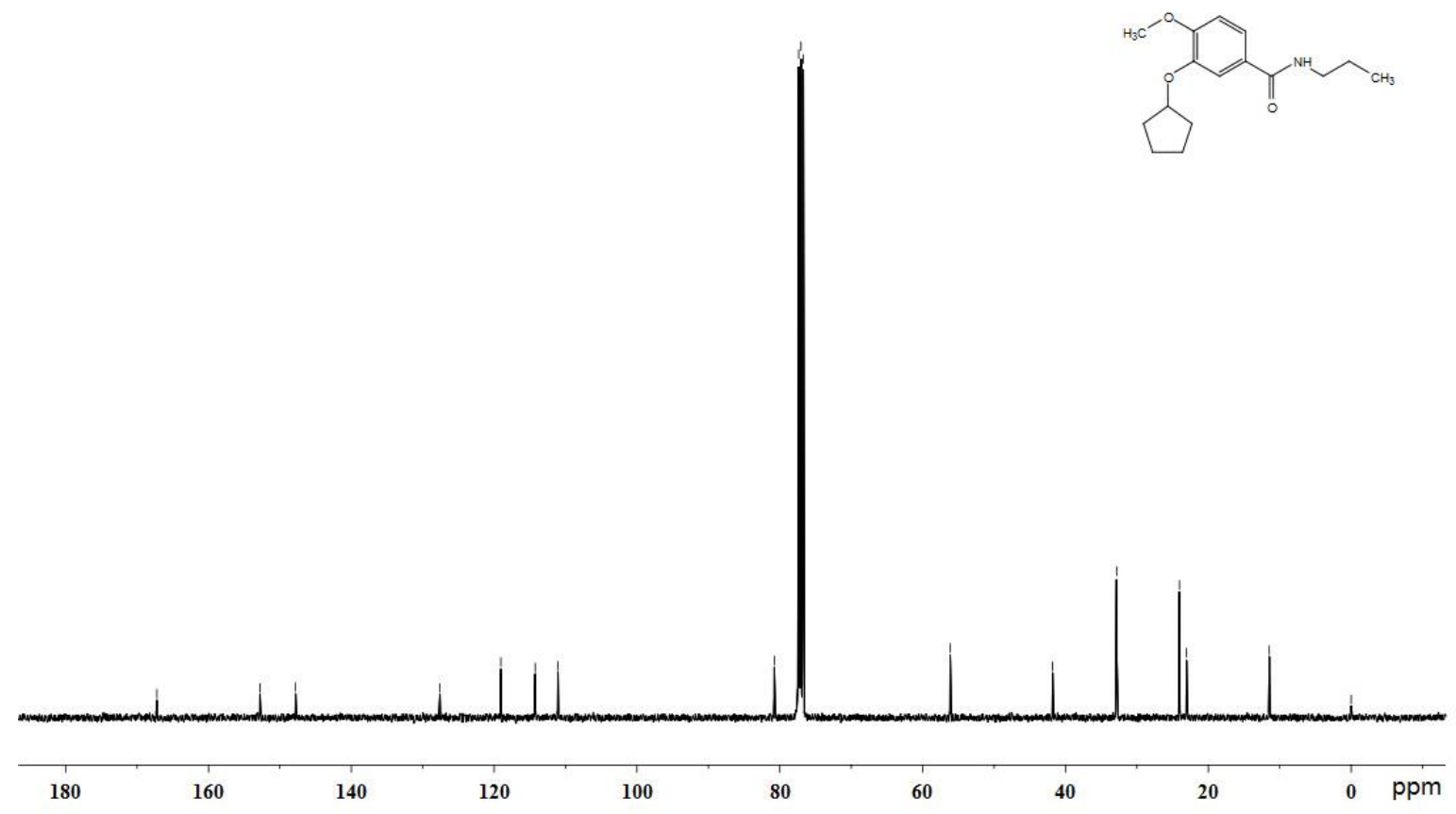

Figure S2. ${ }^{1} \mathrm{H}-\mathrm{NMR}\left(d_{6}\right.$-DMSO, $\left.400 \mathrm{MHz}\right)$ and ${ }^{13} \mathrm{C}-\mathrm{NMR}\left(d_{6}\right.$-DMSO, $\left.101 \mathrm{MHz}\right)$ spectra of compound $\mathbf{1 0 b}$ 


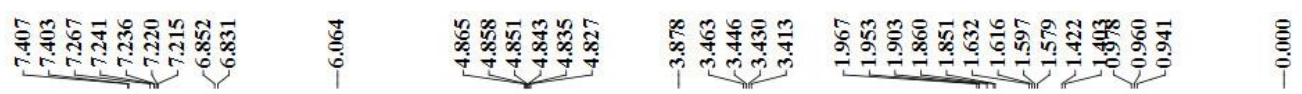
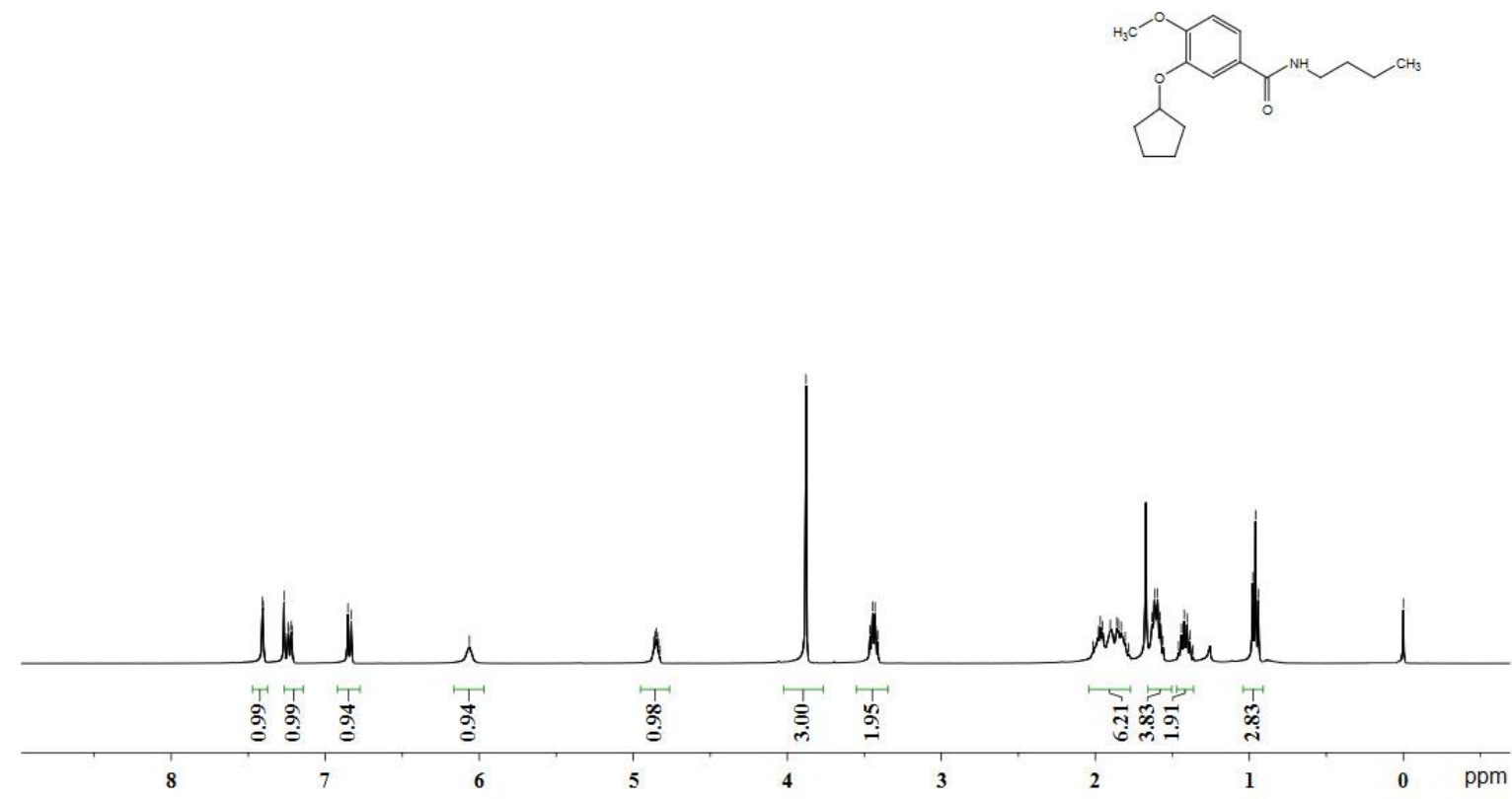

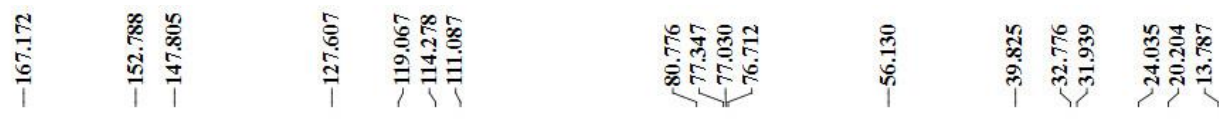

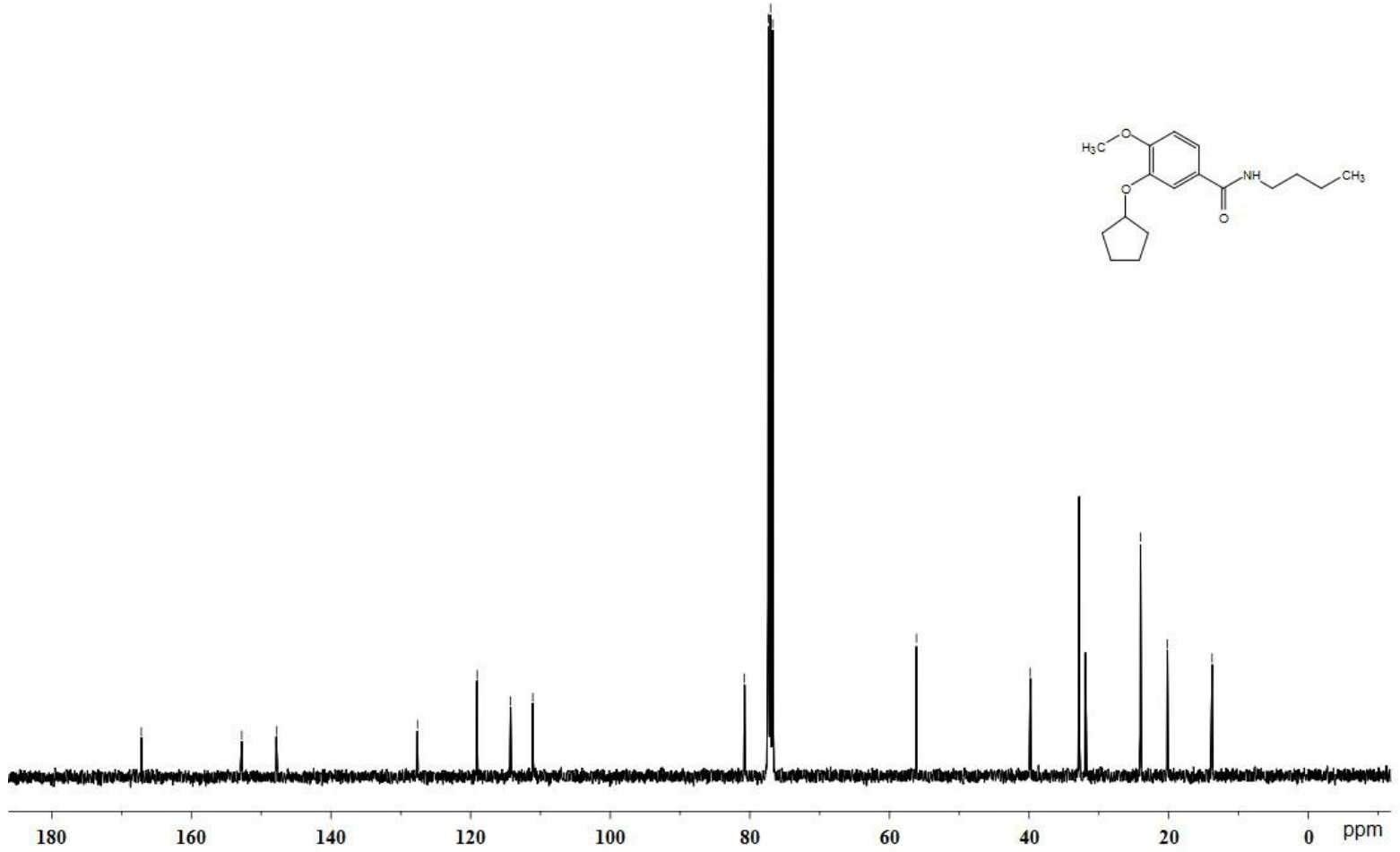

Figure $\mathrm{S} 3 .{ }^{1} \mathrm{H}-\mathrm{NMR}\left(\mathrm{CDCl}_{3,}, 400 \mathrm{MHz}\right)$ and ${ }^{13} \mathrm{C}-\mathrm{NMR}\left(\mathrm{CDCl}_{3}, 101 \mathrm{MHz}\right)$ spectra of compound $10 \mathrm{c}$ 

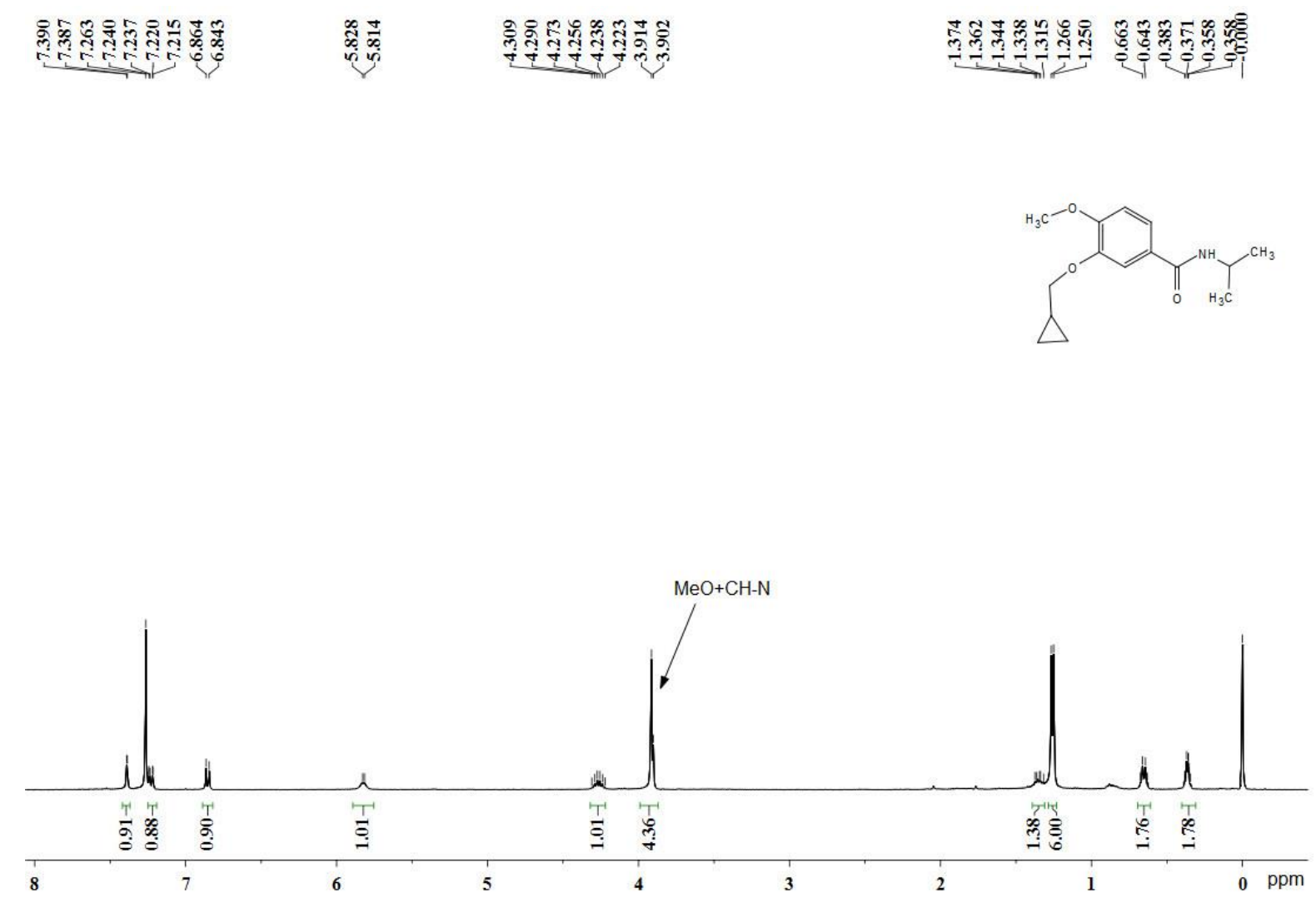

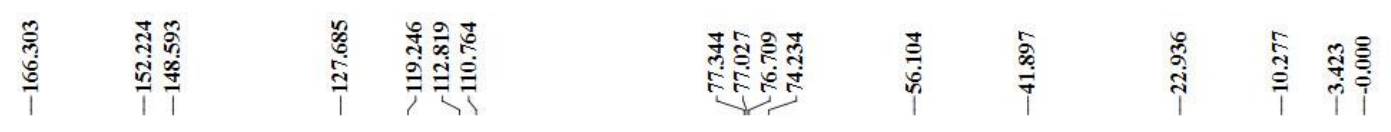

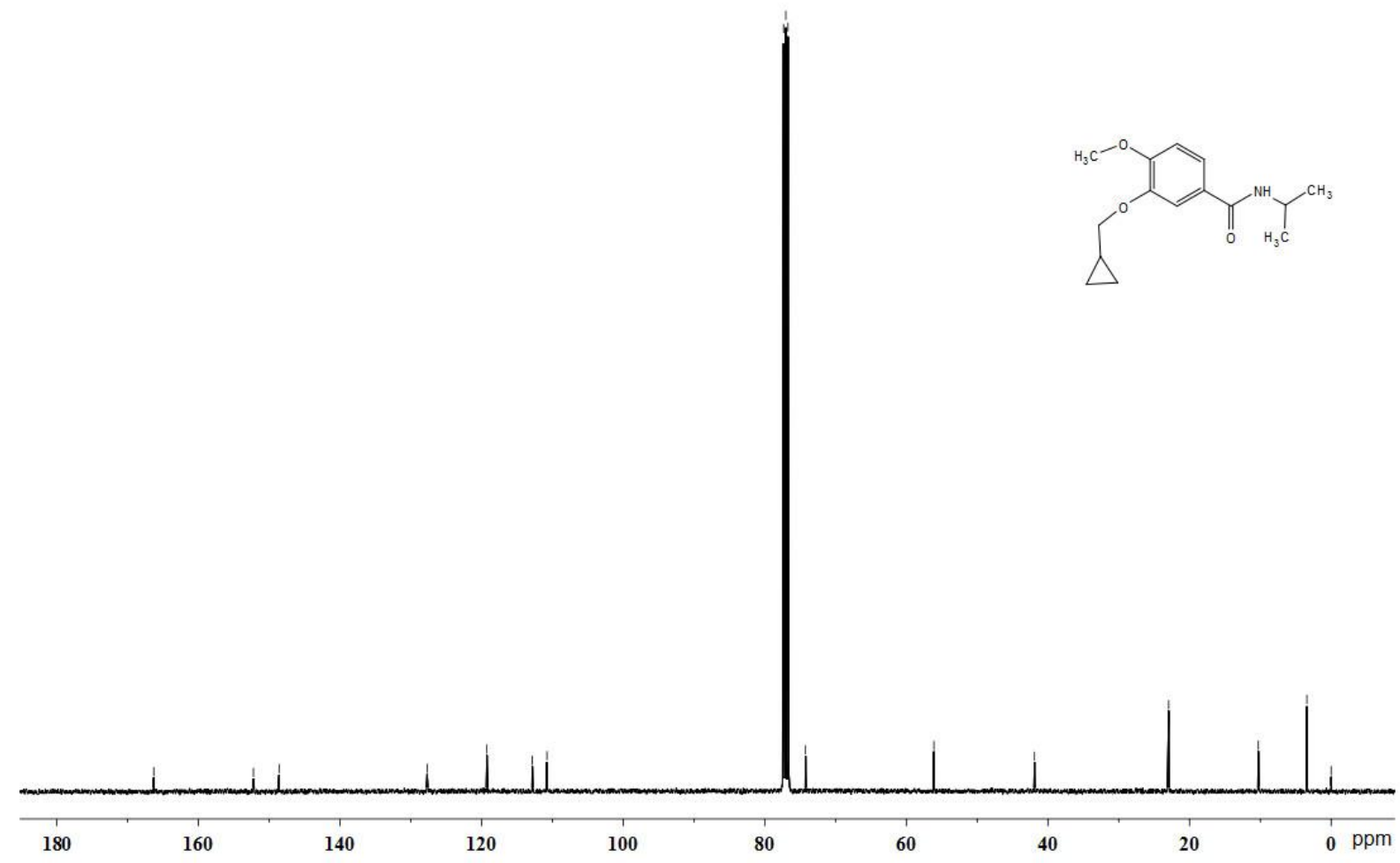

Figure S4. ${ }^{1} \mathrm{H}-\mathrm{NMR}\left(\mathrm{CDCl}_{3}, 400 \mathrm{MHz}\right)$ and ${ }^{13} \mathrm{C}-\mathrm{NMR}\left(\mathrm{CDCl}_{3}, 101 \mathrm{MHz}\right)$ spectra of compound 10d 

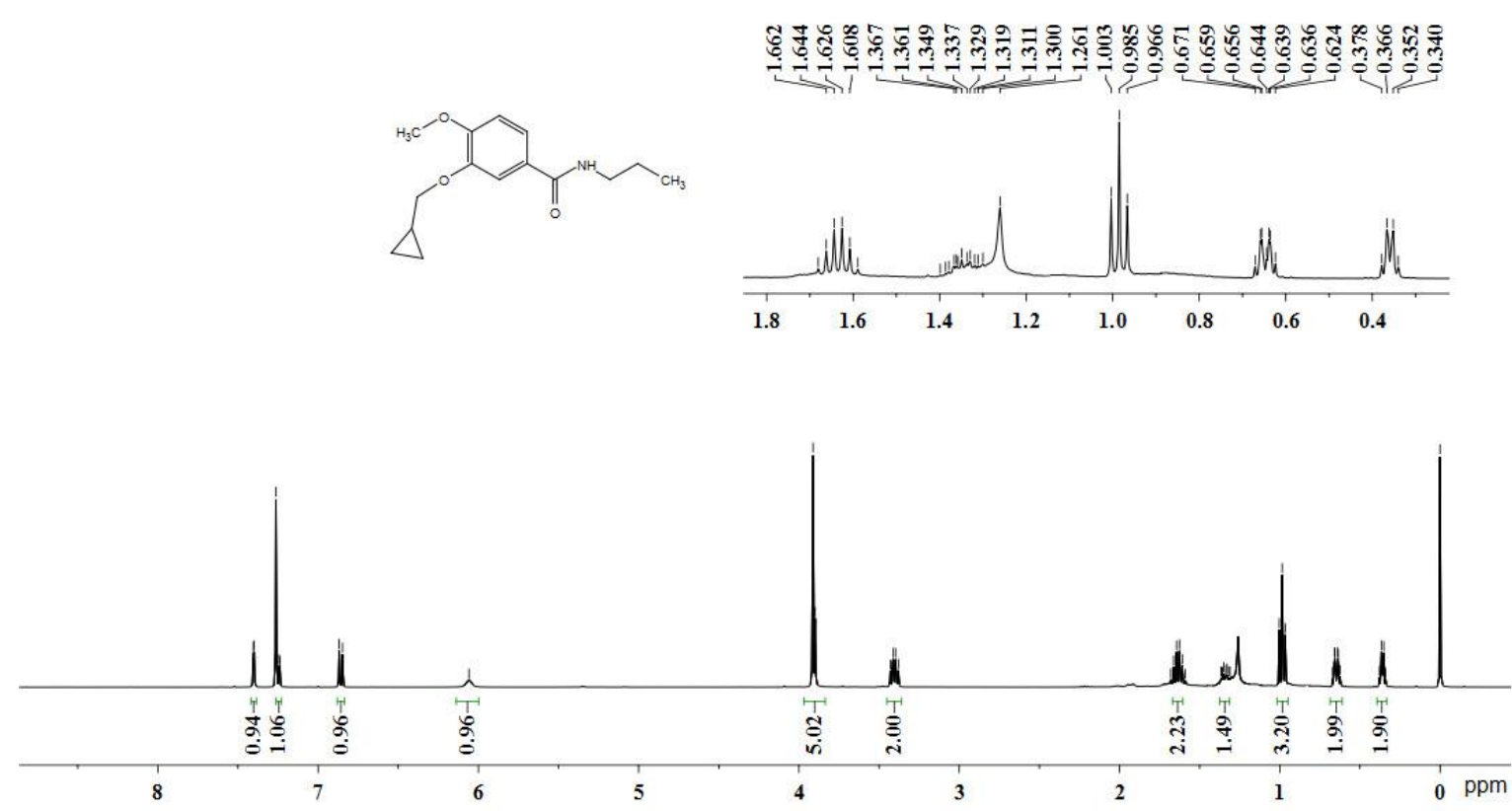

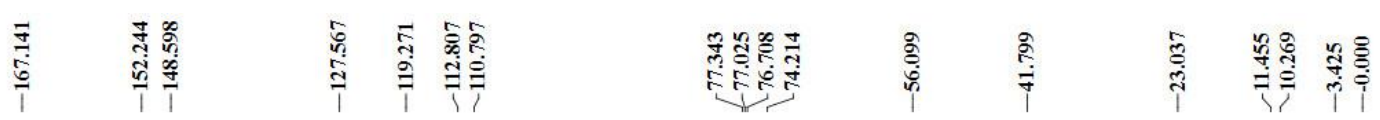

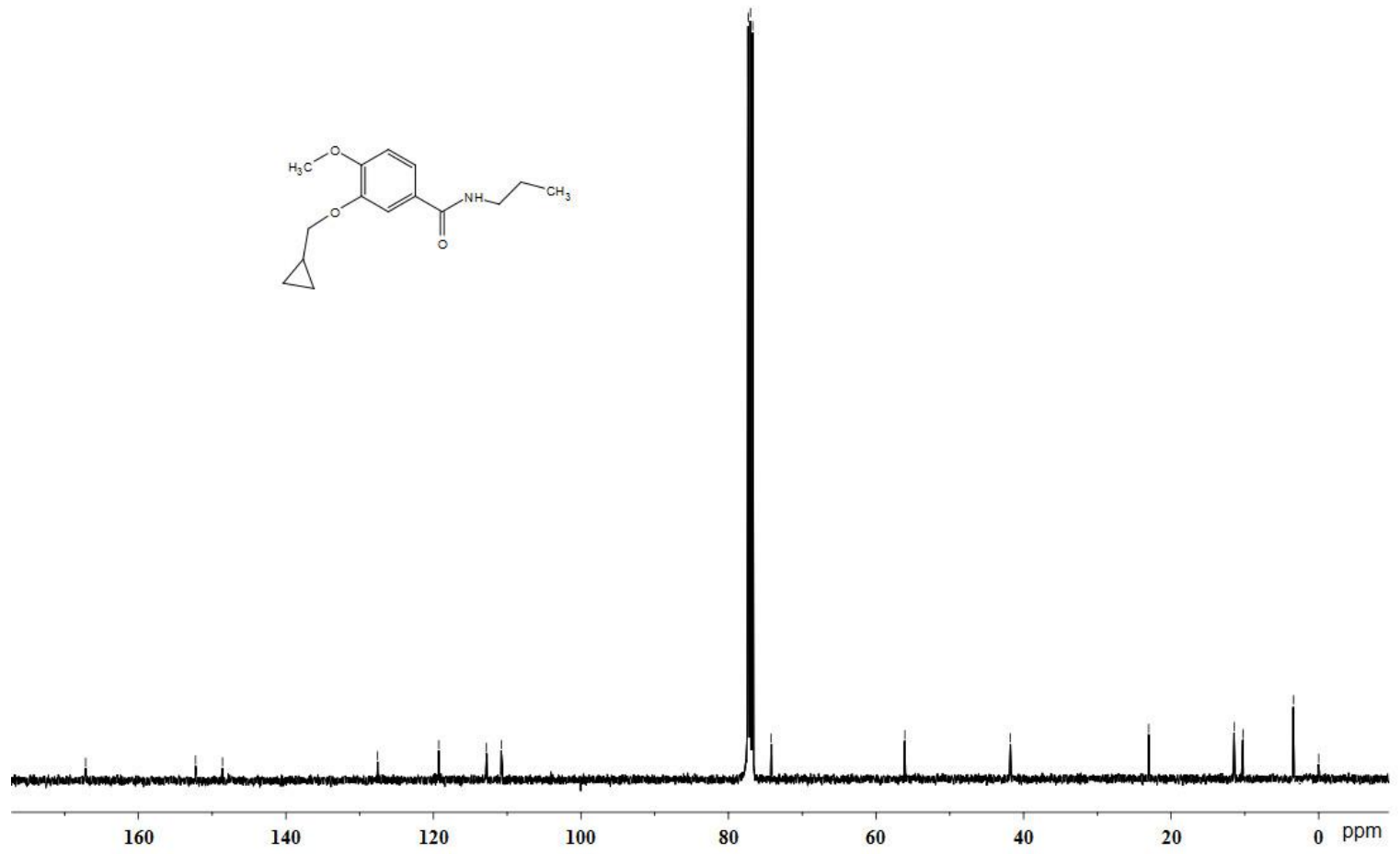

Figure S5. ${ }^{1} \mathrm{H}-\mathrm{NMR}\left(\mathrm{CDCl}_{3}, 400 \mathrm{MHz}\right)$ and ${ }^{13} \mathrm{C}-\mathrm{NMR}\left(\mathrm{CDCl}_{3}, 101 \mathrm{MHz}\right)$ spectra of compound 10e 

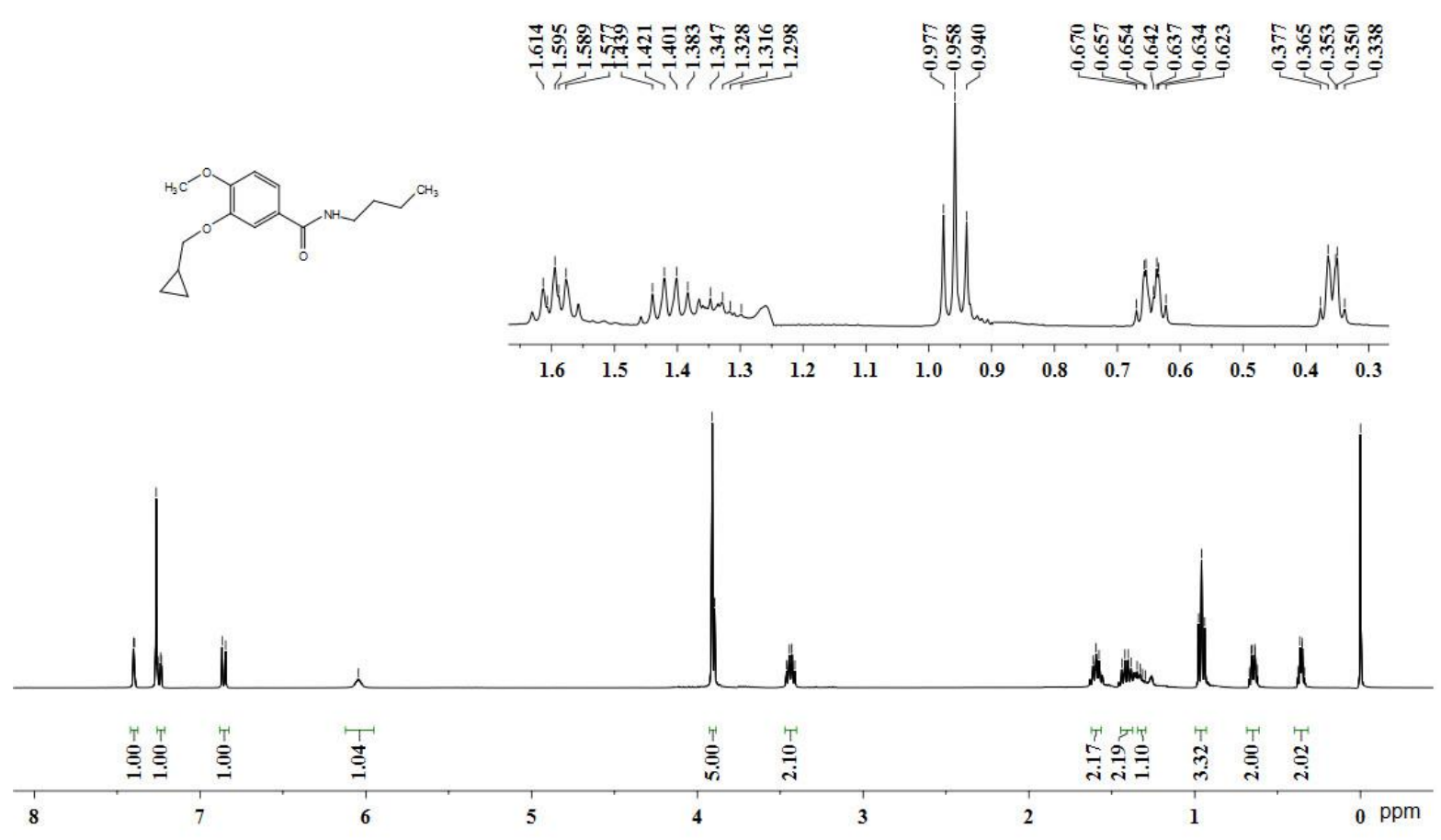

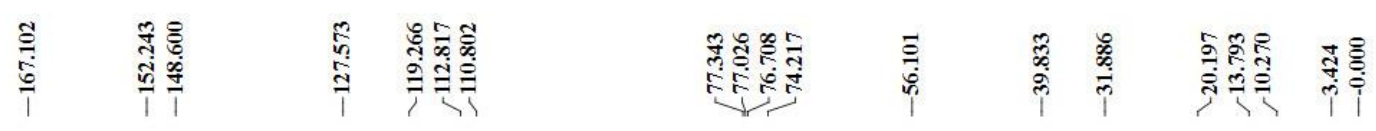

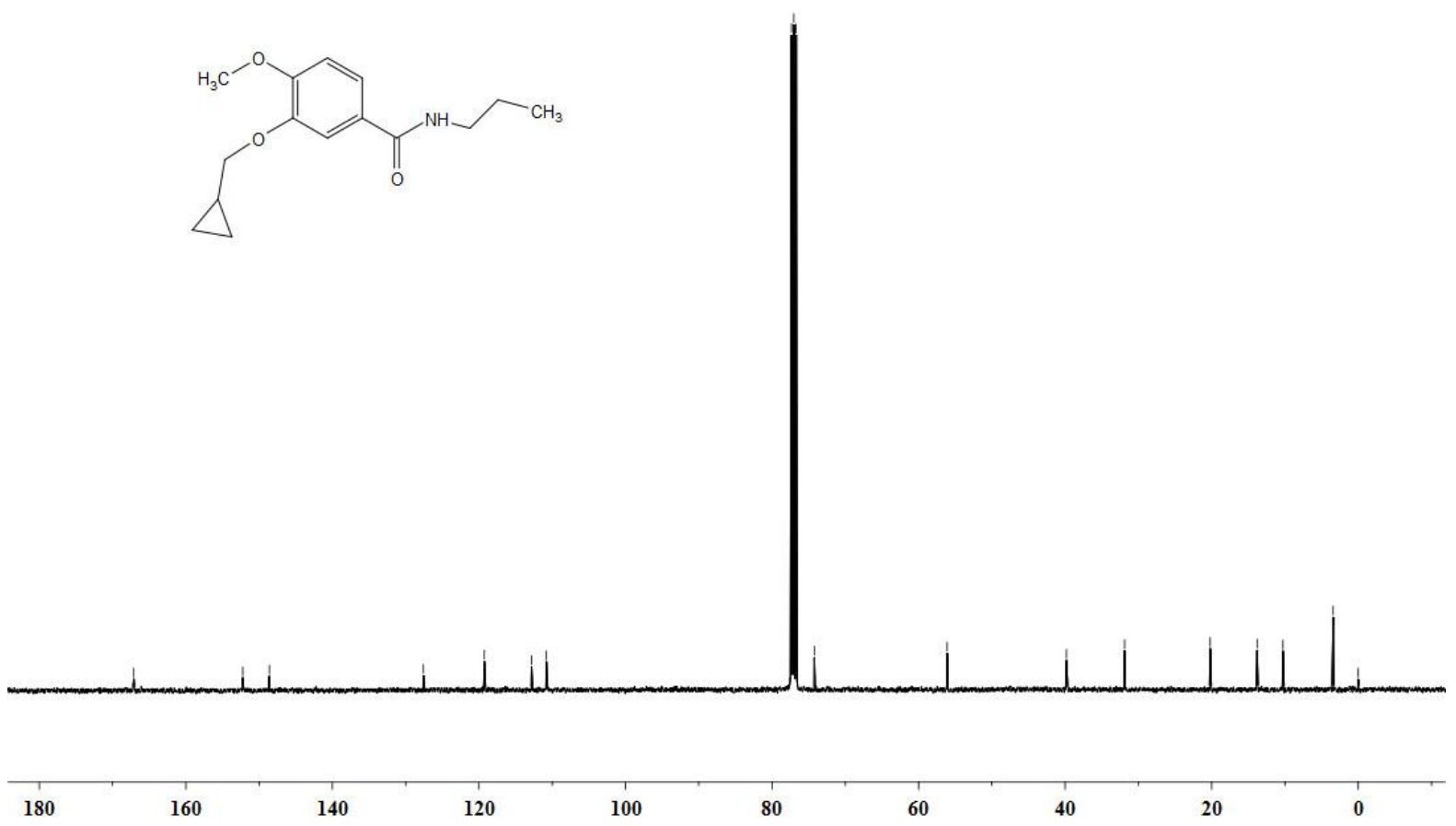

Figure S6. ${ }^{1} \mathrm{H}-\mathrm{NMR}\left(\mathrm{CDCl}_{3,}, 400 \mathrm{MHz}\right)$ and ${ }^{13} \mathrm{C}-\mathrm{NMR}\left(\mathrm{CDCl}_{3}, 101 \mathrm{MHz}\right)$ spectra of compound $\mathbf{1 0 f}$ 


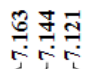

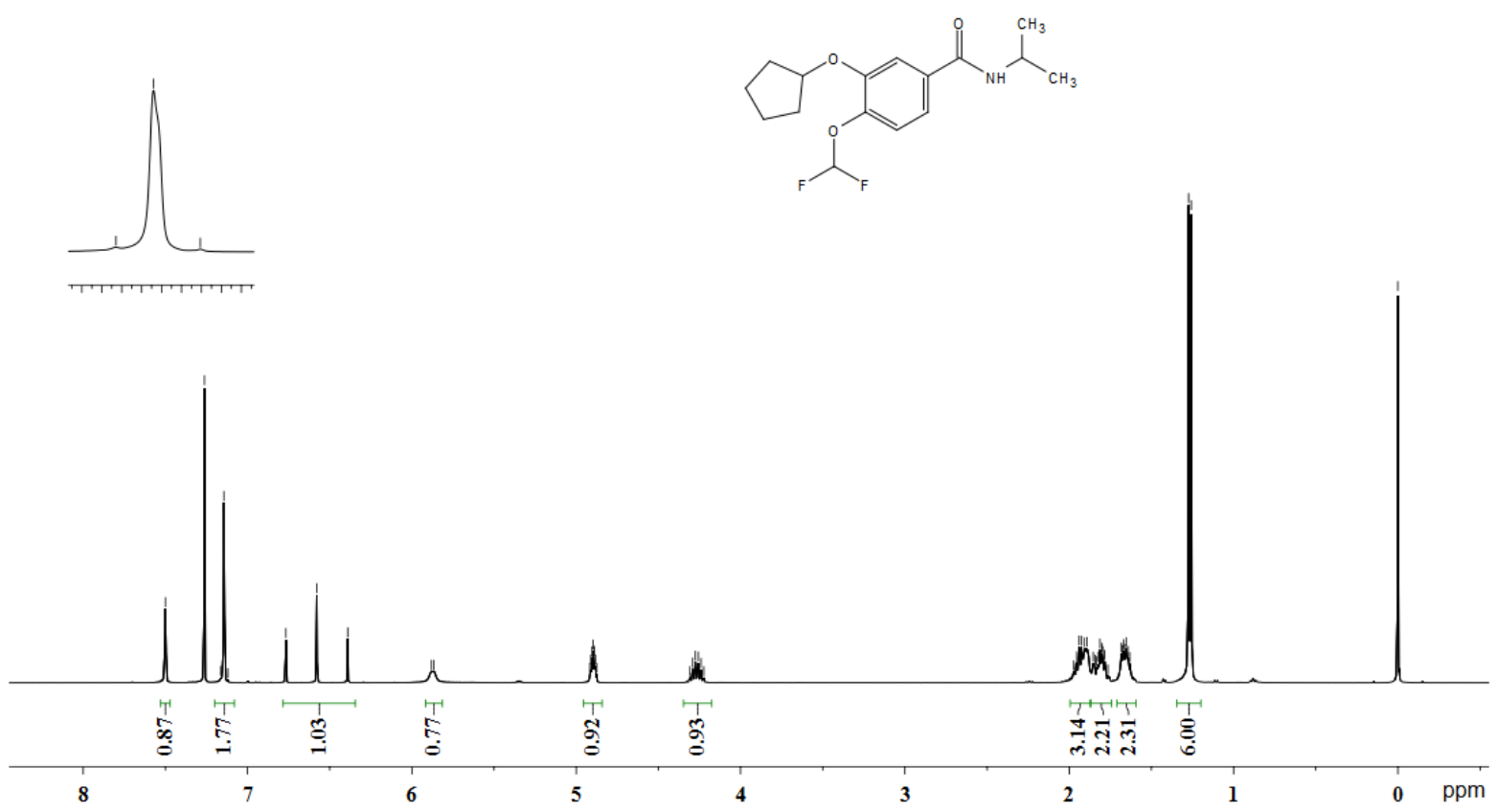

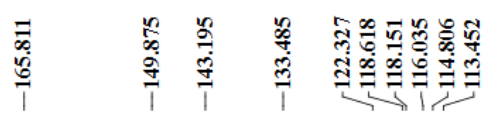

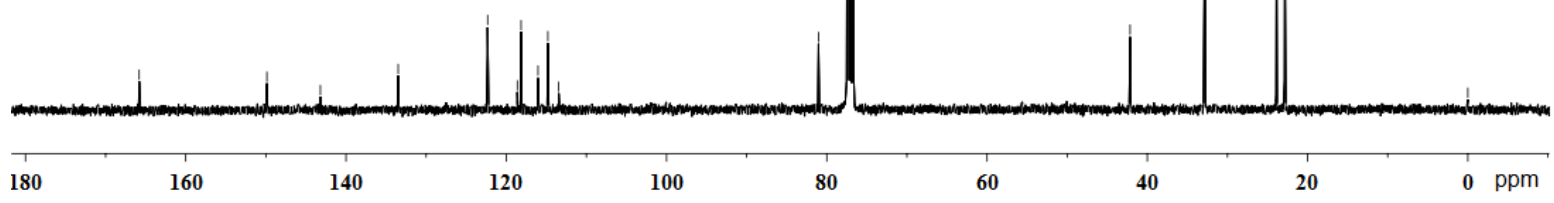

Figure S7. ${ }^{1} \mathrm{H}-\mathrm{NMR}\left(\mathrm{CDCl}_{3}, 400 \mathrm{MHz}\right)$ and ${ }^{13} \mathrm{C}-\mathrm{NMR}\left(\mathrm{CDCl}_{3}, 101 \mathrm{MHz}\right)$ spectra of compound $\mathbf{1 0 g}$ 

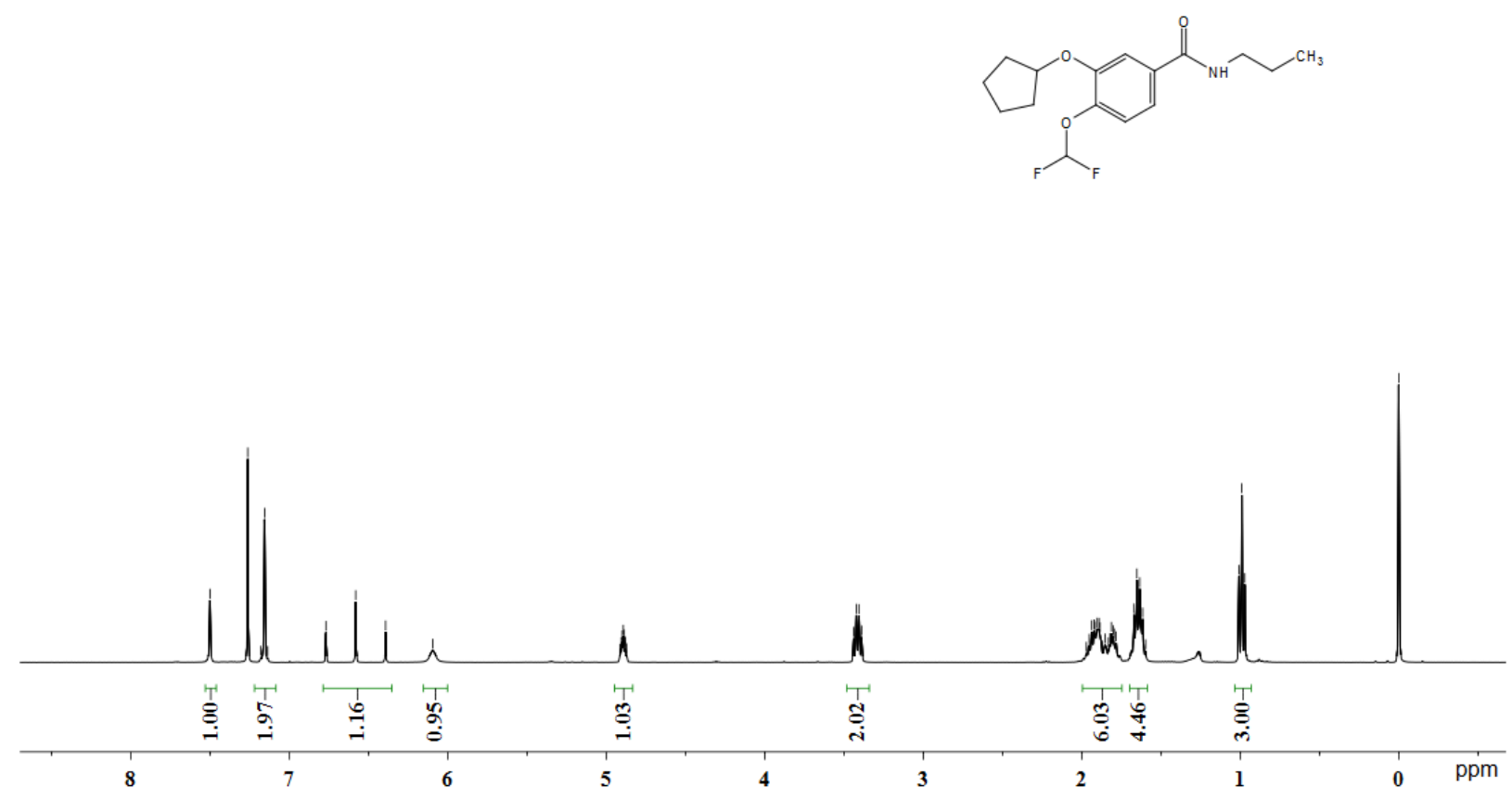

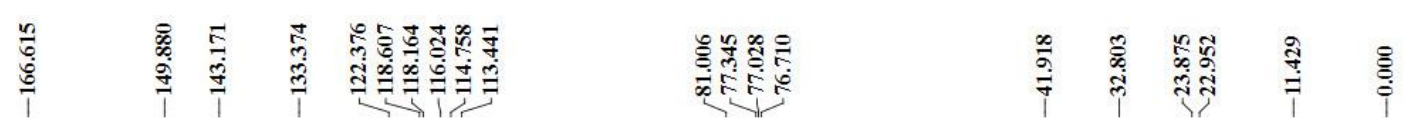

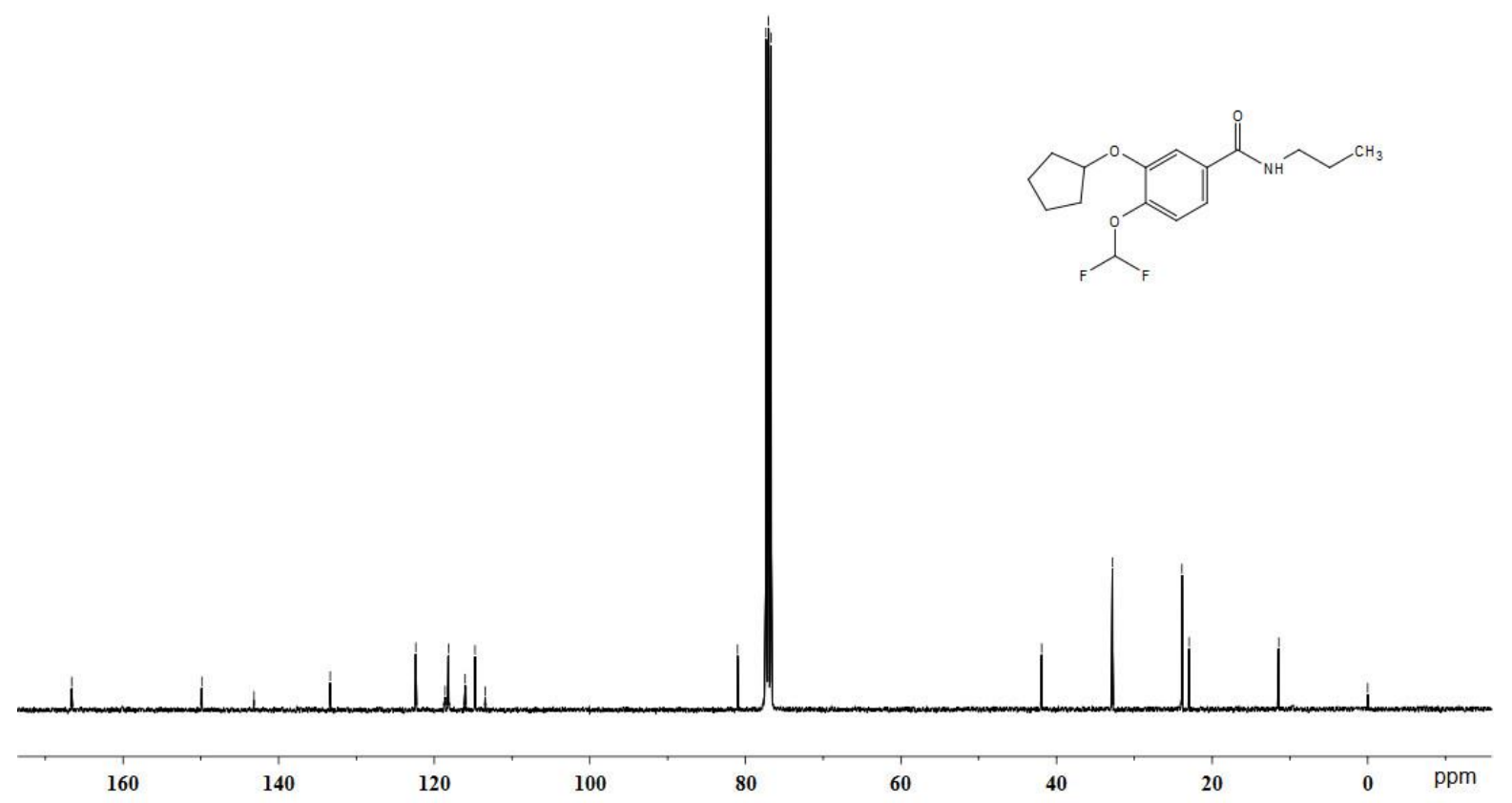

Figure S8. ${ }^{1} \mathrm{H}-\mathrm{NMR}\left(\mathrm{CDCl}_{3}, 400 \mathrm{MHz}\right)$ and ${ }^{13} \mathrm{C}-\mathrm{NMR}\left(\mathrm{CDCl}_{3}, 101 \mathrm{MHz}\right)$ spectra of compound $\mathbf{1 0 h}$ 


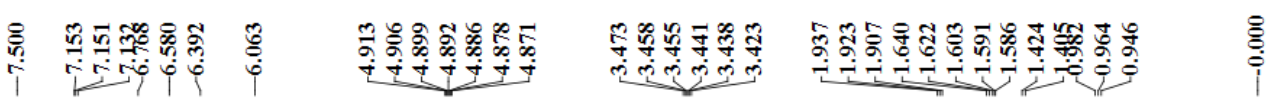
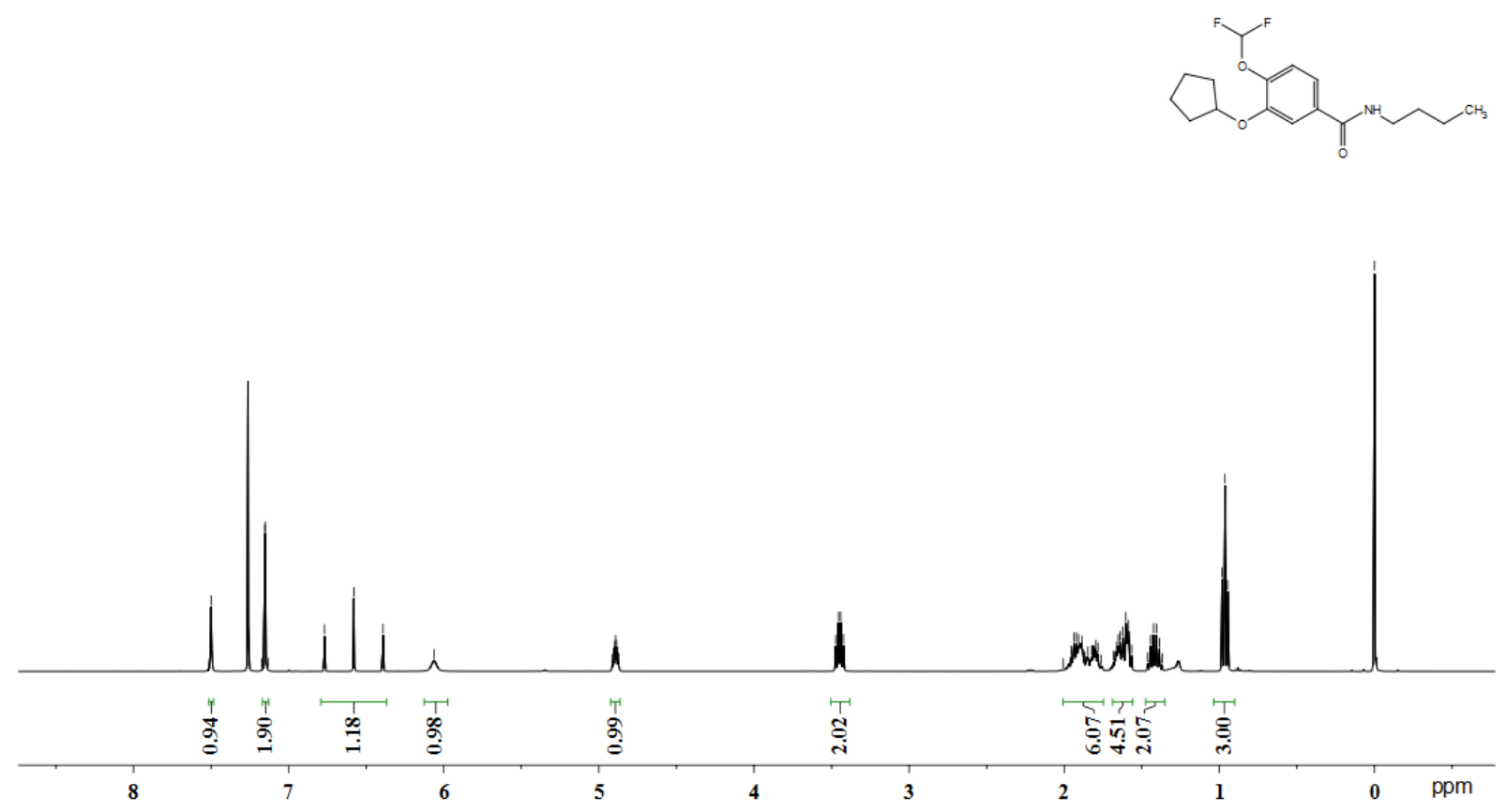

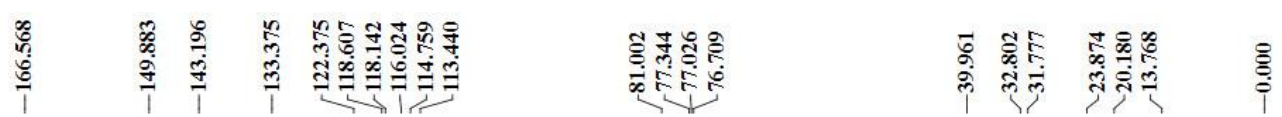

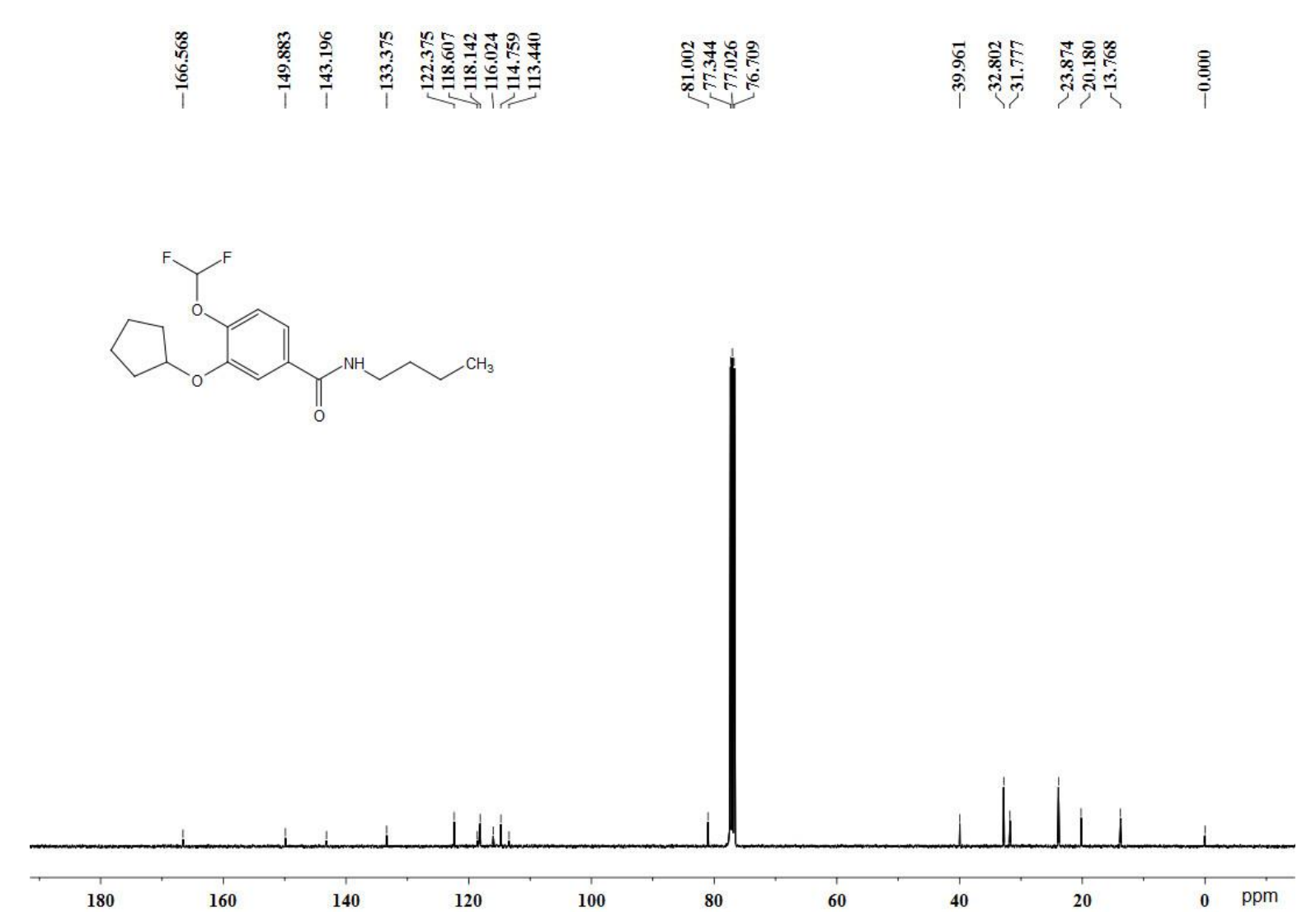

Figure S9. ${ }^{1} \mathrm{H}-\mathrm{NMR}\left(\mathrm{CDCl}_{3}, 400 \mathrm{MHz}\right)$ and ${ }^{13} \mathrm{C}-\mathrm{NMR}\left(\mathrm{CDCl}_{3}, 101 \mathrm{MHz}\right)$ spectra of compound 10i 

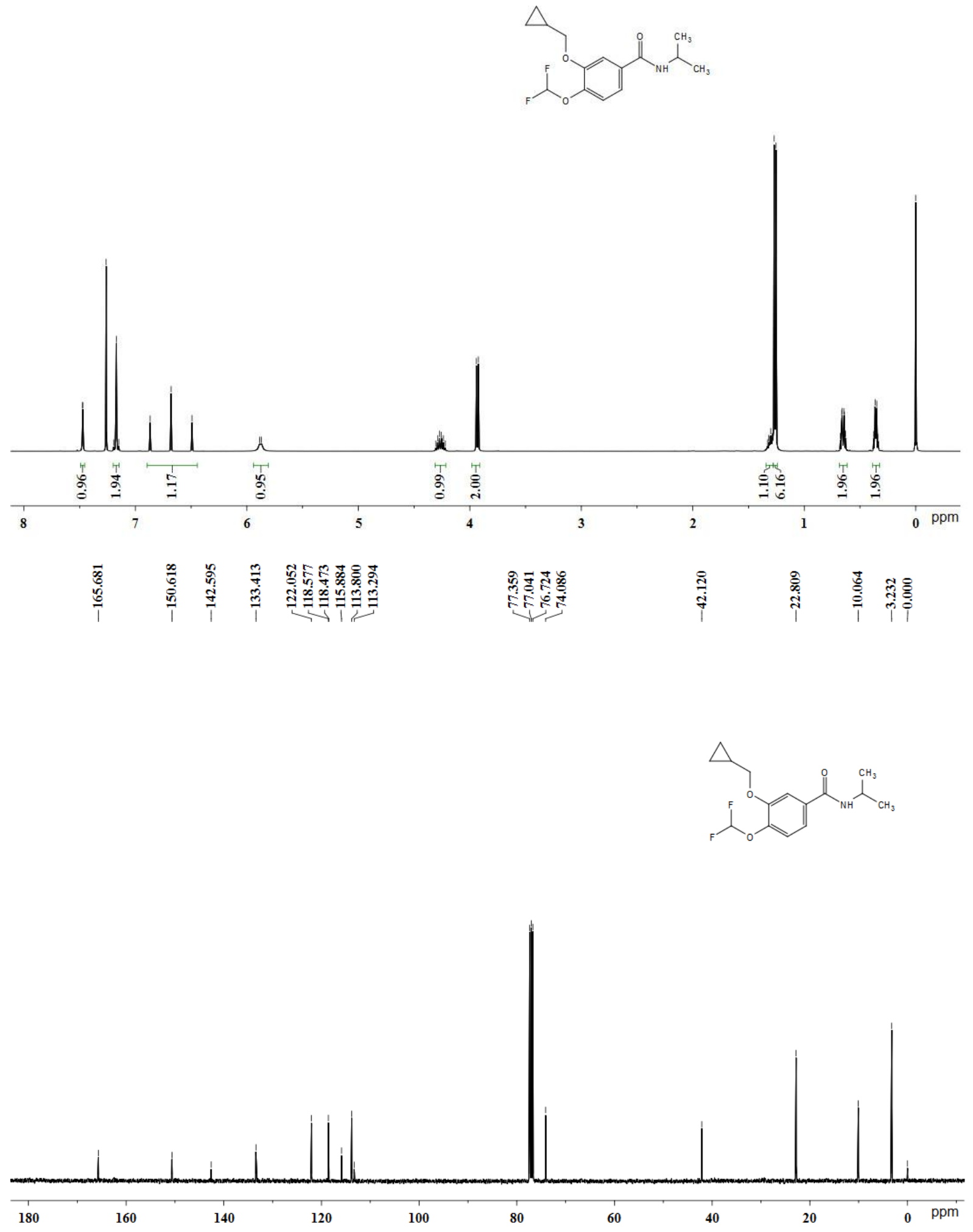

Figure S10. ${ }^{1} \mathrm{H}-\mathrm{NMR}\left(\mathrm{CDCl}_{3}, 400 \mathrm{MHz}\right)$ and ${ }^{13} \mathrm{C}-\mathrm{NMR}\left(\mathrm{CDCl}_{3}, 101 \mathrm{MHz}\right)$ spectra of compound $\mathbf{1 0 j}$ 

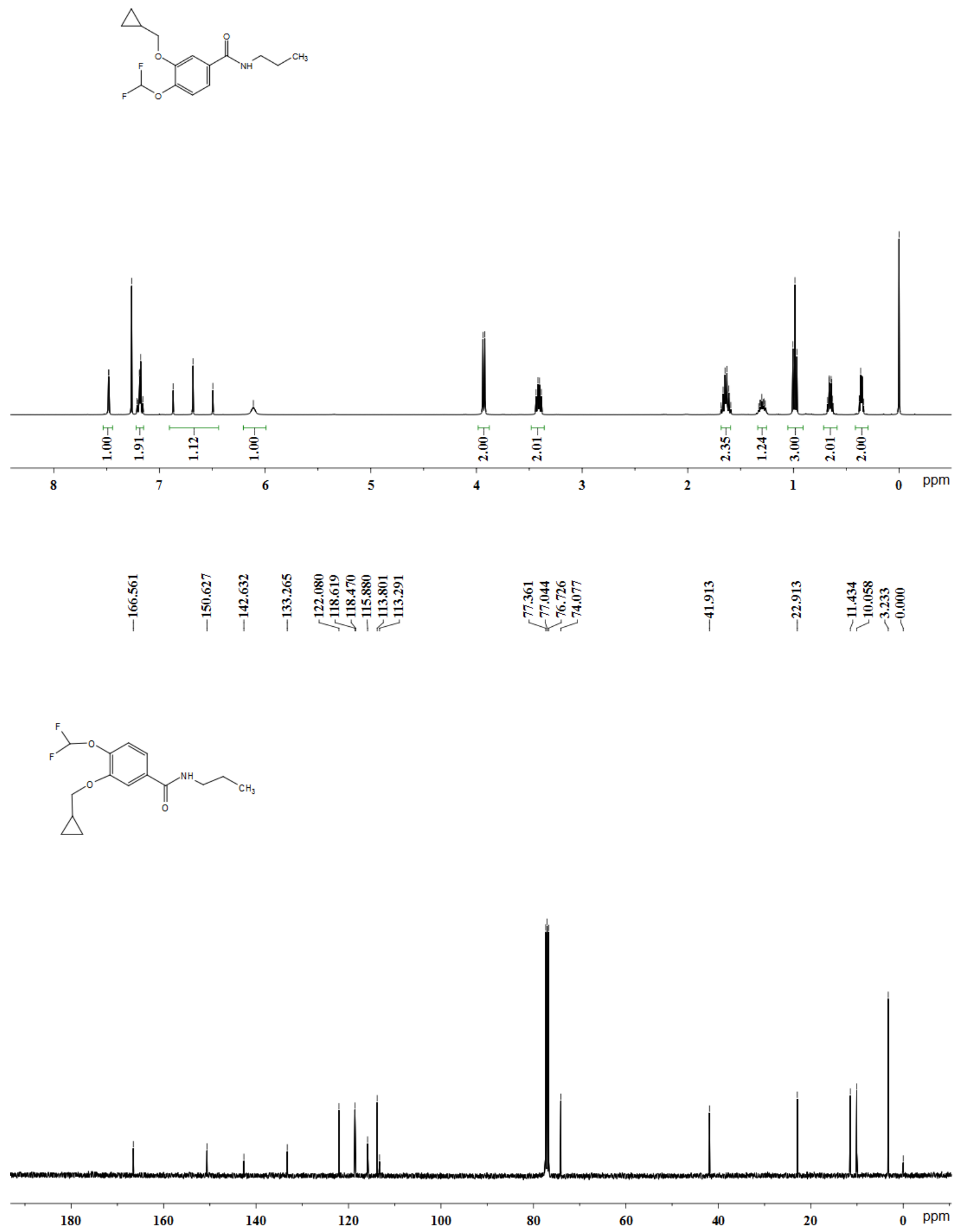

Figure S11. ${ }^{1} \mathrm{H}-\mathrm{NMR}\left(\mathrm{CDCl}_{3}, 400 \mathrm{MHz}\right)$ and ${ }^{13} \mathrm{C}-\mathrm{NMR}\left(\mathrm{CDCl}_{3}, 101 \mathrm{MHz}\right)$ spectra of compound 10k 

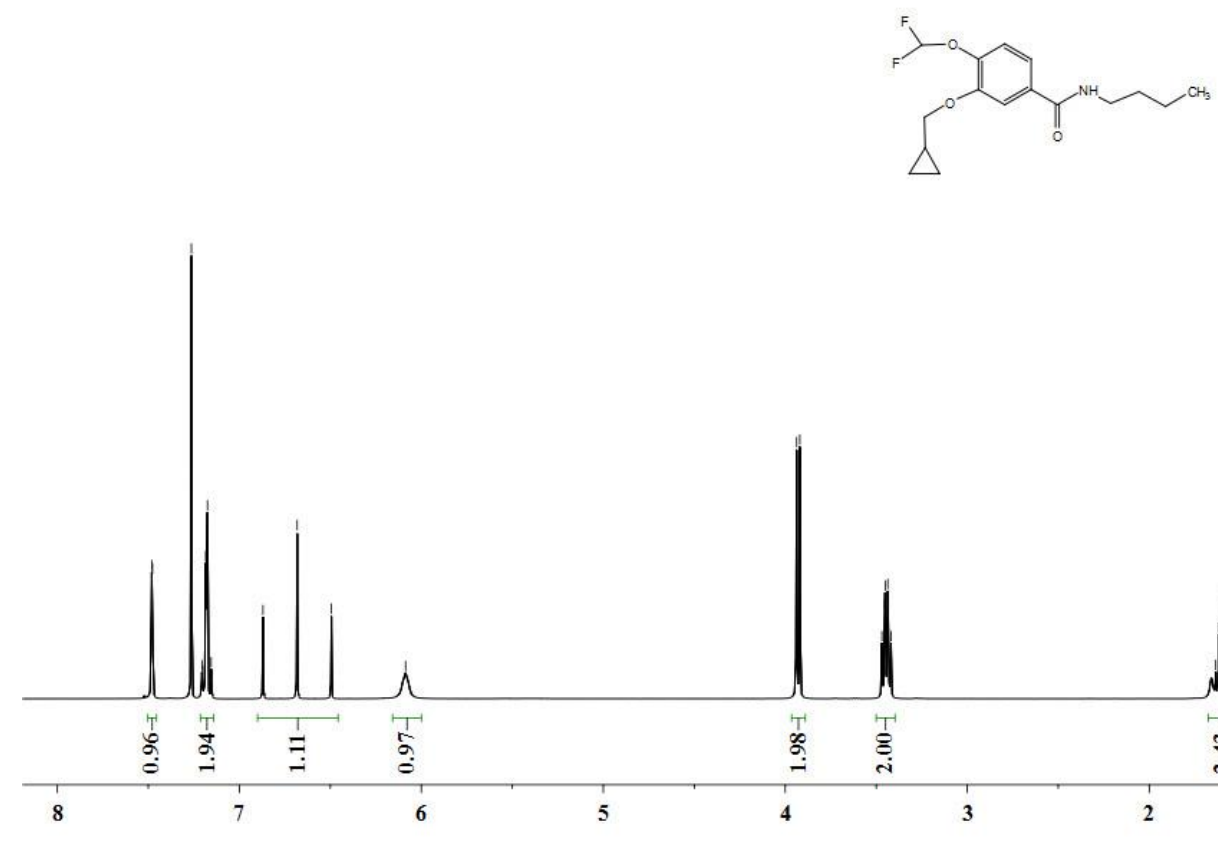

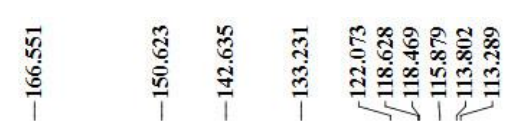
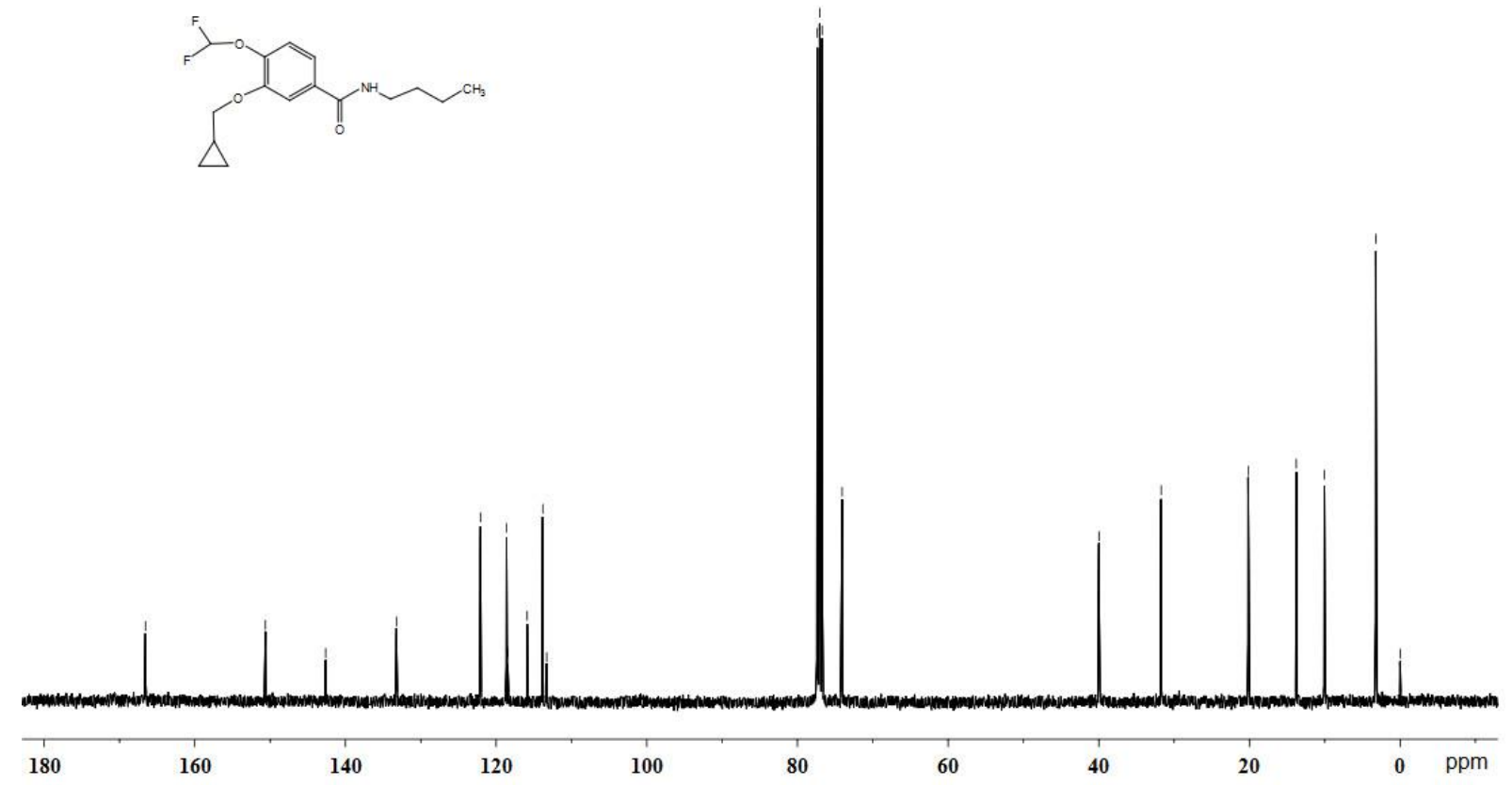

Figure S12. ${ }^{1} \mathrm{H}-\mathrm{NMR}\left(\mathrm{CDCl}_{3}, 400 \mathrm{MHz}\right)$ and ${ }^{13} \mathrm{C}-\mathrm{NMR}\left(\mathrm{CDCl}_{3}, 101 \mathrm{MHz}\right)$ spectra of compound 101 


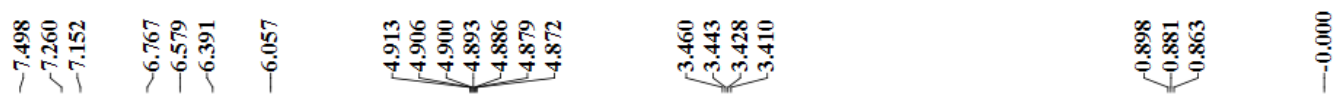
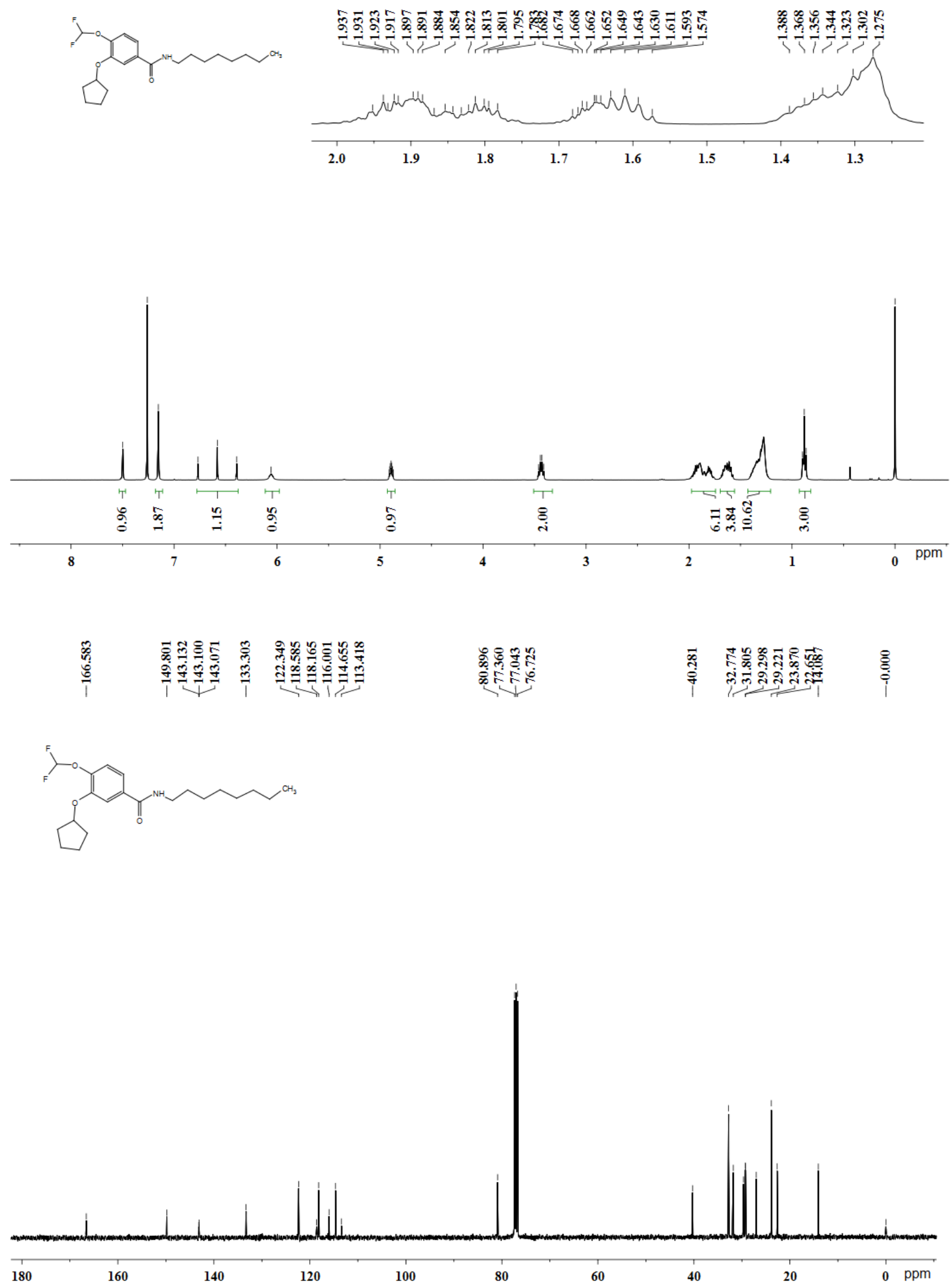

Figure S13. ${ }^{1} \mathrm{H}-\mathrm{NMR}\left(\mathrm{CDCl}_{3}, 400 \mathrm{MHz}\right)$ and ${ }^{13} \mathrm{C}-\mathrm{NMR}\left(\mathrm{CDCl}_{3}, 101 \mathrm{MHz}\right)$ spectra of compound $\mathbf{1 0 m}$ 


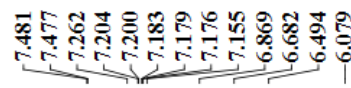

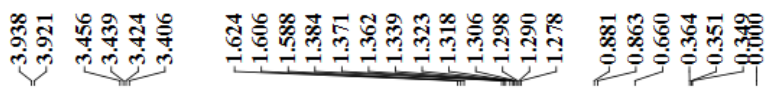

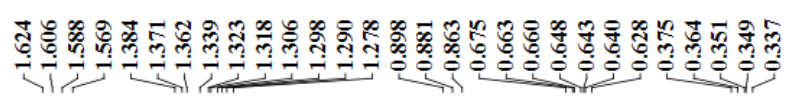
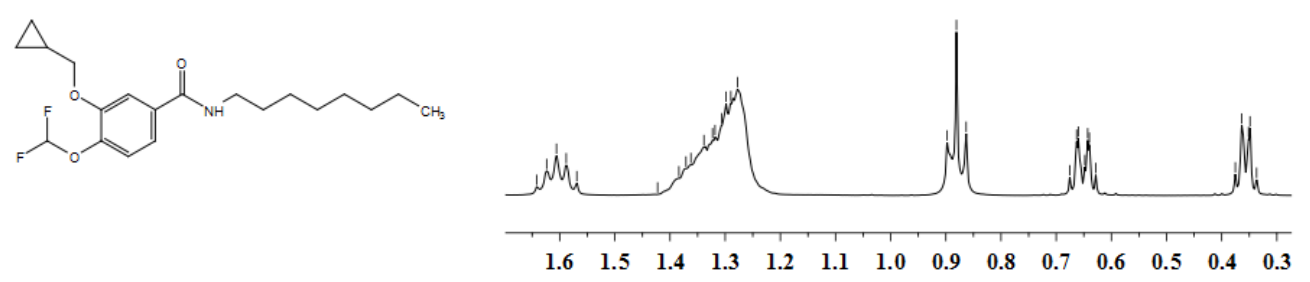
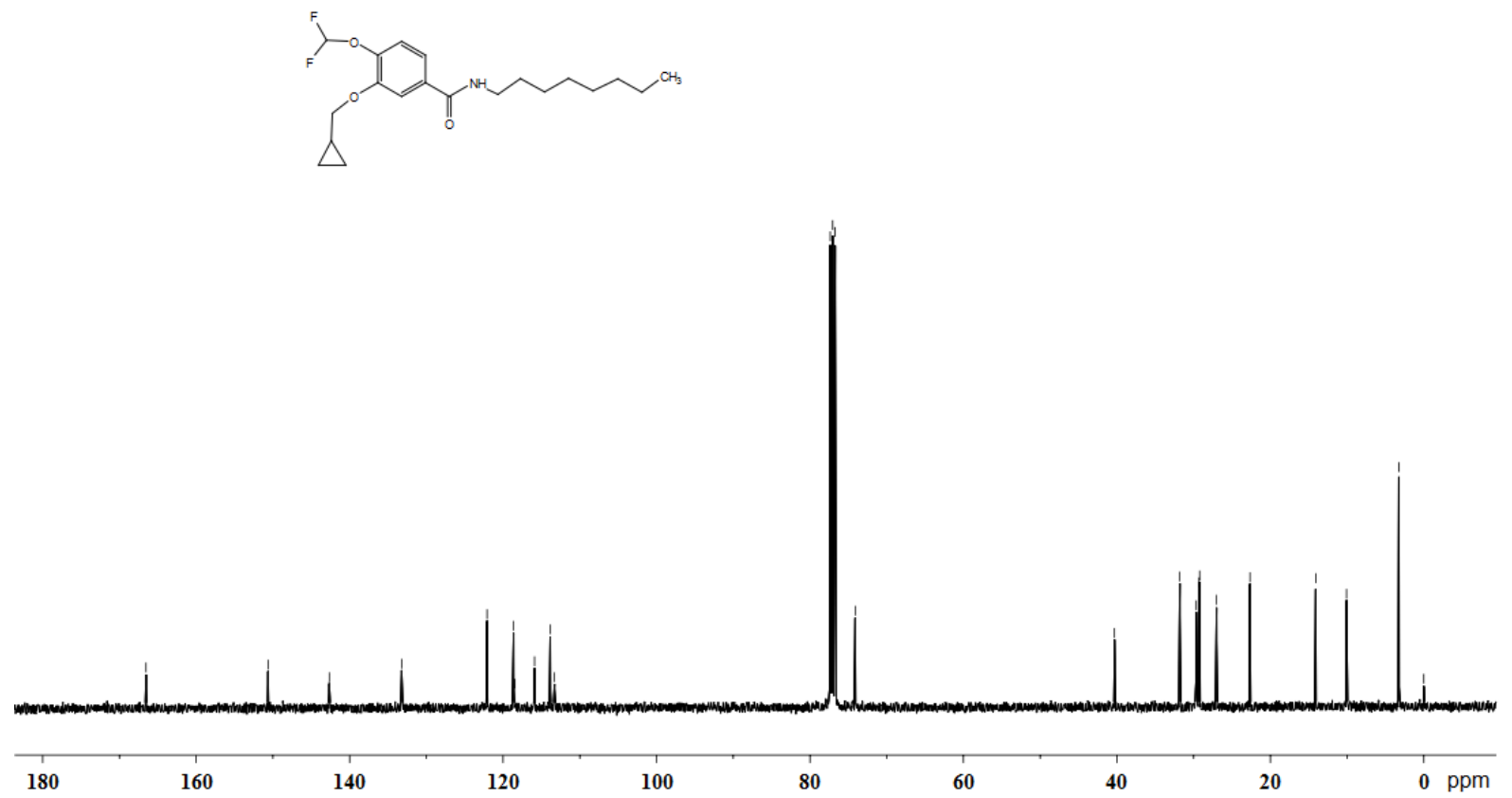

Figure S14. ${ }^{1} \mathrm{H}-\mathrm{NMR}\left(\mathrm{CDCl}_{3,}, 400 \mathrm{MHz}\right)$ and ${ }^{13} \mathrm{C}-\mathrm{NMR}\left(\mathrm{CDCl}_{3}, 101 \mathrm{MHz}\right)$ spectra of compound $10 \mathrm{n}$ 

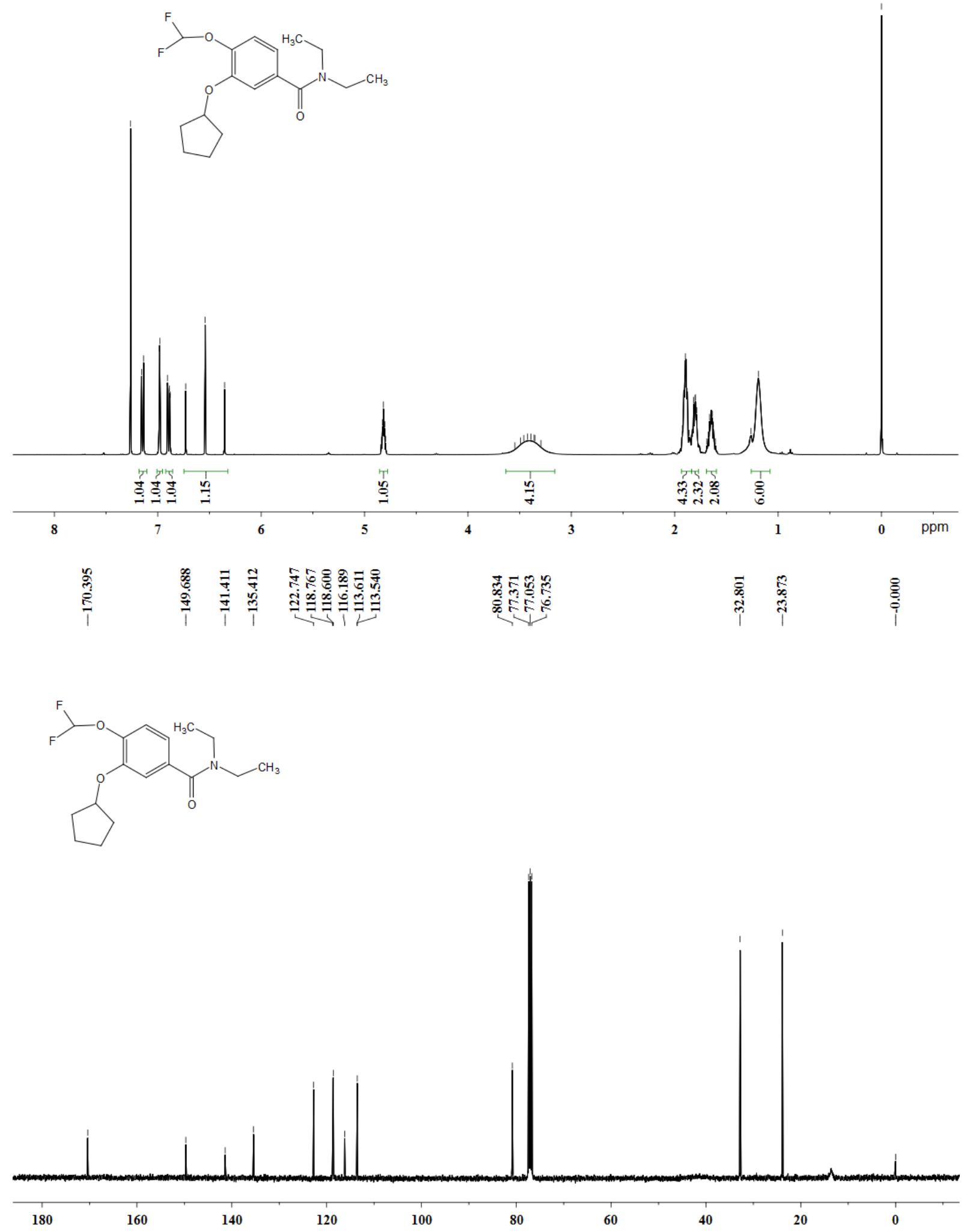

Figure S15. ${ }^{1} \mathrm{H}-\mathrm{NMR}\left(\mathrm{CDCl}_{3}, 400 \mathrm{MHz}\right)$ and ${ }^{13} \mathrm{C}-\mathrm{NMR}\left(\mathrm{CDCl}_{3}, 101 \mathrm{MHz}\right)$ spectra of compound $\mathbf{1 0 0}$ 


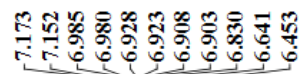

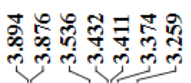

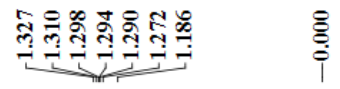<smiles>CCN(CC)C(=O)c1ccc(OC(F)F)c(OCC2CC2)c1</smiles>

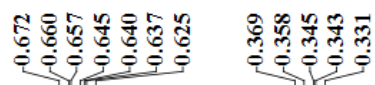
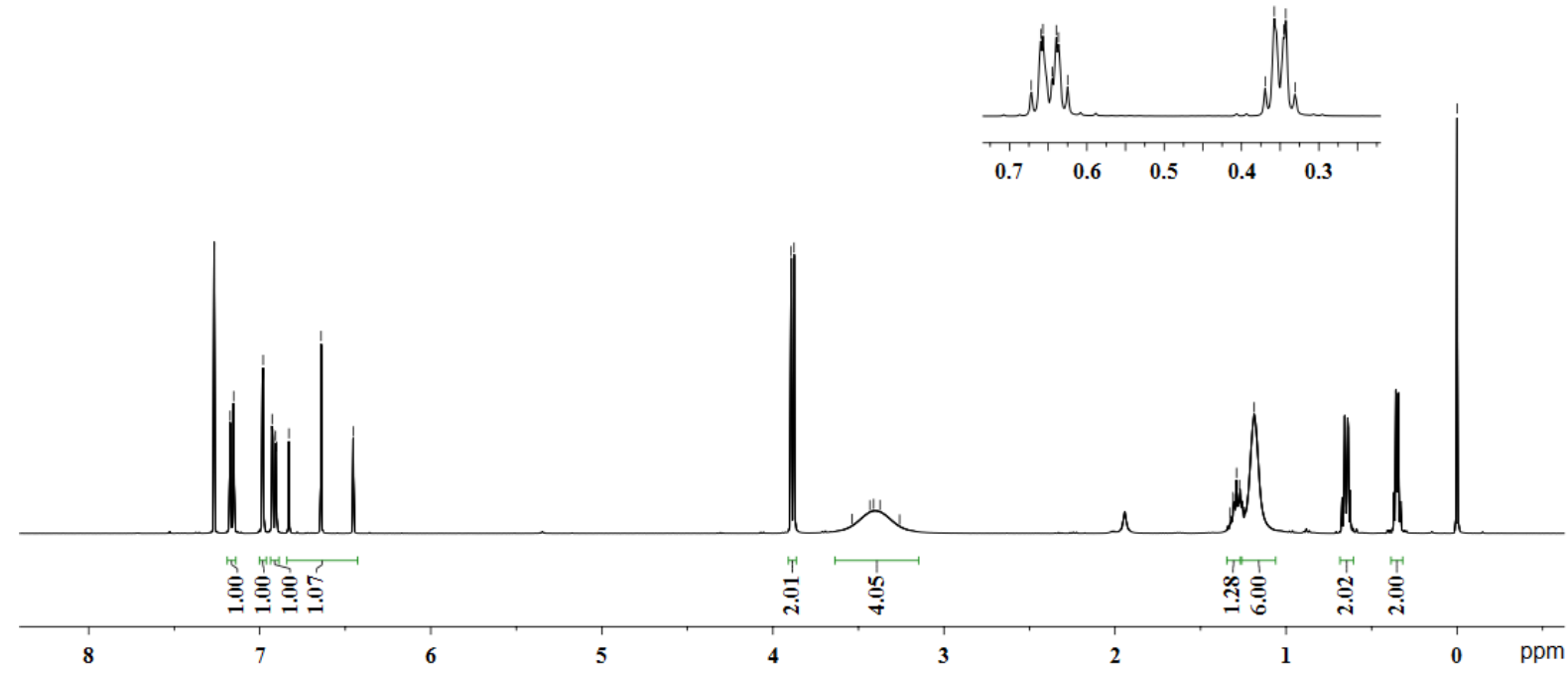

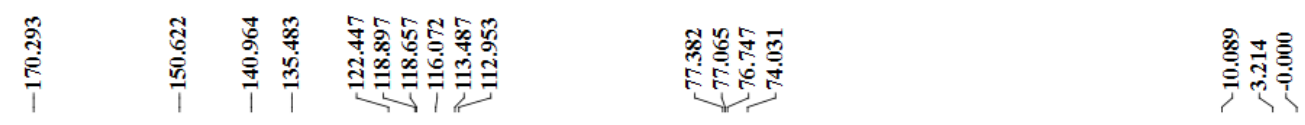
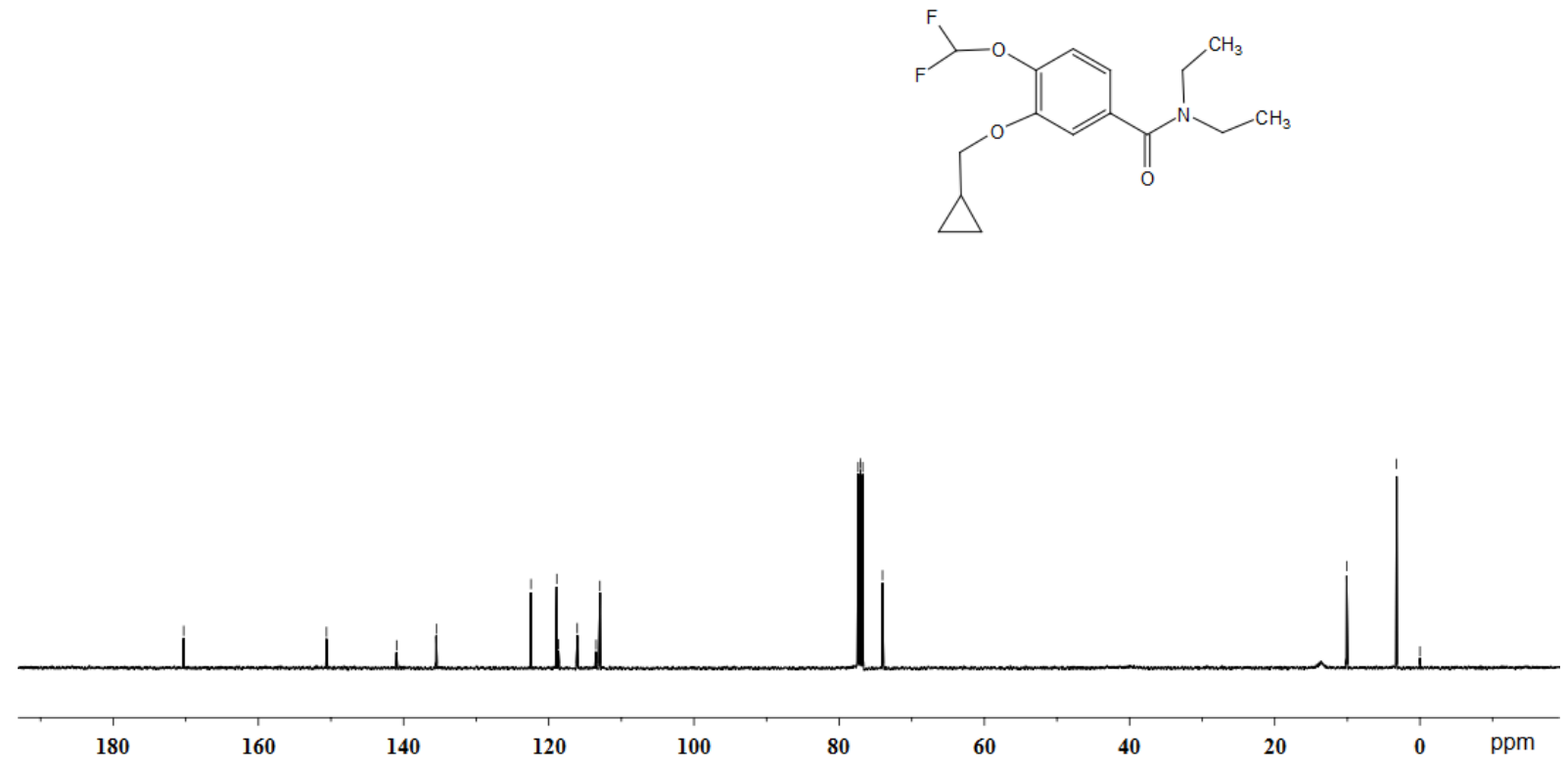

Figure S16. ${ }^{1} \mathrm{H}-\mathrm{NMR}\left(\mathrm{CDCl}_{3}, 400 \mathrm{MHz}\right)$ and ${ }^{13} \mathrm{C}-\mathrm{NMR}\left(\mathrm{CDCl}_{3}, 101 \mathrm{MHz}\right)$ spectra of compound $\mathbf{1 0 p}$ 